\title{
The $p$-canonical basis for Hecke algebras
}

\author{
Lars Thorge Jensen and Geordie Williamson
}

\begin{abstract}
We describe a positive characteristic analogue of the Kazhdan-Lusztig basis of the Hecke algebra of a crystallographic Coxeter system and investigate some of its properties. Using Soergel calculus we describe an algorithm to calculate this basis. We outline some known or expected applications in modular representation theory. We conclude by giving several examples.
\end{abstract}

\section{Introduction}

One consequence of categorification is that it often leads to a "canonical" basis in the structure being categorified. Canonical bases have numerous remarkable applications in representation theory and beyond.

The first examples of canonical bases are the Kazhdan-Lusztig basis of Hecke algebras and the canonical basis of quantum groups. These bases have geometric origins, arising as the shadow of a sheaf (an intersection cohomology sheaf) on a variety. That one should obtain interesting bases in this way is, in some sense, predicted by Grothendieck's function-sheaf correspondence. ${ }^{1}$

More recently, considerable progress has been made via algebraic approaches to categorification. Here the canonical basis arises as the

2000 Mathematics Subject Classification. Primary 20C20, Secondary 20C08, 17B10, 14M15.

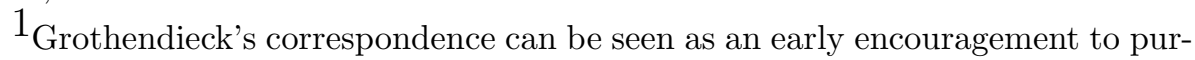
sue categorification, by replacing a function (an object of a set) by a sheaf or $D$-module (an object of a category). Despite many more examples illustrating the power of this correspondence (e.g. Lusztig's theory of character sheaves, the geometric Langlands program, ...), it still seems remarkable that Grothendieck's prophecy really works!

(C)0000 (copyright holder) 
shadow of a simple or indecomposable projective module over an algebra. For example, the canonical basis of the quantum group is realized in simply laced type as the classes in the Grothendieck group of indecomposable projective modules for KLR algebras (see [VV11]). Similarly, the Kazhdan-Lusztig basis arises as the classes of indecomposable Soergel bimodules (see [EW14]). The passage between algebra and geometry is made by realizing an algebra as an extension algebra of geometric origin [Rou12, VV11, Soe01].

The canonical basis (or rather, the base change matrix between the canonical basis and a standard basis) usually records multiplicities in categories of interest in representation theory, which are defined over a field of characteristic 0 . This is reflected by the choice of coefficients of characteristic zero (both in the geometric and algebraic settings). Usually it is only via appeal to powerful geometric theorems (e.g. [Del80, BBD82, Sai90, dCM09]) that one has an algorithm to compute the canonical basis (however see [EW14]).

There are several questions in modular representation theory where certain multiplicities are expected to be given in terms of a canonical basis. Famous examples include Lusztig's conjecture for simple rational modules for algebraic groups [Lus80], the LLT conjecture for representations of Hecke algebras at roots of unity [LLT96, Ari96], and the James conjecture on representations of the symmetric group [Jam90]. These conjectures predict that the situation in characteristic 0 agrees with the one in characteristic $p \gg 0$, sometimes state an explicit bound on the "bad" primes to exclude, but usually aren't so bold as to make any statement about what happens for small $p$.

However recent work by several authors suggests a different way of thinking about this problem. Namely, in important examples there exists a " $p$-canonical basis" for each choice of prime number $p$. The $p$ canonical basis has the same indexing set as the usual canonical basis, and each of its elements agrees with the corresponding element of the canonical basis for large enough $p$, but may differ from it for small $p$. One hopes that the $p$-canonical basis gives the correct answer in representation theory in characteristic $p$. Examples of this phenomenon include:

(1) The work of Grojnowski, Ariki and others [Gro99, Ari96] identifying the Grothendieck group of the category of representations of all symmetric groups with the basic representation of an affine Lie algebra. Here the $p$-canonical basis is defined via the classes of indecomposable projective modules, and hence the $p$-canonical basis contains deep information in 
modular representation essentially by definition. (It is from [Gro99] that we first learnt the term $p$-canonical basis.)

(2) The work of Soergel [Soe00], connecting certain multiplicities in the rational representation theory of algebraic groups with indecomposable summands of Bott-Samelson sheaves. Subsequent work (e.g. [JMW14a, WB12]) shows that Soergel's results may be restated as giving these multiplicities in terms of coefficients of the p-canonical basis of the Hecke algebra of the finite Weyl group.

(3) A recent conjecture of Riche and the second author, which predicts that the characters of indecomposable tilting modules for algebraic groups should be given in terms of coefficients of the $p$-canonical basis in the anti-spherical module of the affine Weyl group. By Schur-Weyl duality this conjecture implies that all decomposition numbers for symmetric groups occur as coefficients in this basis. In contrast to (1), this equivalence is not "by definition", and might lead to more efficient algorithms. In recent work, Riche and the second author have confirmed their conjecture for the general linear group.

The above approach breaks problems in modular representation theory into two subproblems. First one should connect the problem in representation theory to the $p$-canonical basis, and then one should calculate the $p$-canonical basis. In general the second step seems to be extremely difficult, and it is possibly too optimistic to expect an answer in general [Wil13]. However at the very least this approach has the potential to unify questions in modular representation theory (just as different questions may have answers given by the same KazhdanLusztig polynomials). Also, there are combinatorial constraints on the $p$-canonical basis which make the situation very rigid, and might merit further investigation.

1.1. In this paper we recall the definition of and study the $p$ canonical basis of the Hecke algebra of a crystallographic Coxeter system $(W, S)$ (see [Wil12] where the definition appears in print for the first time). To motivate its definition, we first recall how the KazhdanLusztig basis arises from categorification. In the introduction, let us assume that $W$ is the Weyl group of a complex reductive group $G$ with a maximal torus $T$. Let $B \subset G$ denote the Borel subgroup corresponding to the simple reflections $S \subset W$.

The Hecke algebra $\mathcal{H}$ of $(W, S)$ has two (essentially equivalent) categorifications, often referred to loosely as the Hecke category: 
(1) Geometric: The additive, monoidal (under convolution) category of $B$-biequivariant semi-simple complexes on $G$ : the full subcategory of the equivariant derived category $D_{B \times B}^{b}(G, \mathbb{Q})$ consisting of direct sums of shifts of equivariant intersection cohomology complexes.

(2) Algebraic: The monoidal category of Soergel bimodules: a certain full subcategory of the monoidal category of graded $R$ bimodules, where $R$ denotes the regular functions on the Lie algebra of the maximal torus $T$.

In the first setting, the Kazhdan-Lusztig basis arises as the graded dimensions of stalks of the intersection cohomology complexes (see [KL80]). In the second case, the Kazhdan-Lusztig basis is realized as the characters of the indecomposable self-dual Soergel bimodules (see [Soe98, EW14]).

In [EW13] the monoidal category of Soergel bimodules is described by generators and relations, following earlier work by Elias [Eli13], Elias-Khovanov [EK10] and Libedinsky [Lib10] (we recall this description in detail below). The upshot is that there exists a graded monoidal category $\mathbf{H}$ which is defined over the integers, and whose extension of scalars to $\mathbb{Q}$ is equivalent to Soergel bimodules. Hence one can think of $\mathbf{H}$ as an integral form of the Hecke category. For any field $k$ we can consider the extension of scalars ${ }^{k} \mathbf{H}$ to $k$ and it is proved in [EW13] that one has an canonical "character" isomorphism of $\mathbb{Z}\left[v, v^{-1}\right]$-algebras

$$
\operatorname{ch}:\left[{ }^{k} \mathbf{H}\right] \stackrel{\sim}{\longrightarrow} \mathcal{H}
$$

between the split Grothendieck group of ${ }^{k} \mathbf{H}$ and the Hecke algebra. Hence for any field ${ }^{k} \mathbf{H}$ provides a categorification of the Hecke algebra. ${ }^{2}$ (Note that while the coefficients of $\mathbf{H}$ change, the Grothendieck group is always the same Hecke algebra over $\mathbb{Z}\left[v, v^{-1}\right]$.)

In [EW 13] the indecomposable objects of ${ }^{k} \mathbf{H}$ are classified, following Soergel's classification of the indecomposable Soergel bimodules in [Soe07]. It turns out that for all $w \in W$ there exists an indecomposable object ${ }^{k} B_{w} \in{ }^{k} \mathbf{H}$, and that any indecomposable object is isomorphic to a grading shift of ${ }^{k} B_{w}$ for some $w \in W$. The $p$-canonical basis is defined as the character of this indecomposable object:

$$
{ }^{p} \underline{H}_{w}:=\operatorname{ch}\left({ }^{k} B_{w}\right)
$$

where $p$ denotes the characteristic of $k$. From basic properties of ${ }^{k} \mathbf{H}$ it is easy to see that $\left\{{ }^{p} \underline{H}_{w} \mid w \in W\right\}$ is a basis for the Hecke algebra

\footnotetext{
${ }^{2}$ There is a minor additional technical assumption if the characteristic of $k$ is 2. We ignore this point in the introduction.
} 
which only depends on the characteristic of $k$, and that its structure constants are positive (see Proposition 4.2). Moreover, because ${ }^{\mathbb{Q}} \mathbf{H}$ is equivalent to Soergel bimodules with $\mathbb{Q}$-coefficients, $\left\{{ }^{0} \underline{H}_{w} \mid w \in W\right\}$ is the Kazhdan-Lusztig basis.

1.2. In this subsection we outline the connection between the $p$ canonical basis and parity sheaves on (affine) flag varieties. The reader unfamiliar with affine flag varieties may keep the important case of a (finite) flag variety in mind.

To any based root datum we can associate a connected, reductive algebraic group scheme $G$ over $\mathbb{Z}$ with Borel subgroup $B \subseteq G$ and maximal torus $T \subseteq B \subseteq G$. In the finite case, let $X$ denote the $\mathbb{C}$-points of the flag variety $\mathcal{F l}:=G / B$ with its classical metric topology.

In the affine setting, define the loop group $L G$ (resp. positive loop group $L^{+} G$ ) of $G$ as the $\mathbb{Z}$-functor given by $R \mapsto G(R((t)))$ (resp. $R \mapsto$ $G(R[[t]]))$. Denote by $I$ the Iwahori subgroup given by the inverse image of $B$ under the morphism $L^{+} G \rightarrow G$ induced by $t \mapsto 0$. In this case we define $X$ to be the $\mathbb{C}$-points of the affine flag variety $\mathcal{F} l_{a}:=L G / I$ viewed as ind-variety (see [Gör10] for more information).

In both settings we have an (Iwahori)-Bruhat decomposition expressing the corresponding flag variety as a disjoint union of left $B(\mathbb{C})$ (resp. $I(\mathbb{C})$ )-orbits indexed by the (extended affine) Weyl group. The closure relation is given by the Bruhat order. Note that the affine flag variety is isomorphic as an ind-variety to a suitable disjoint union of Kac-Moody flag varieties (see e.g. [Kum02]).

Fix a field $k$ of coefficients. We may consider $D_{H}^{b}(X)$ the $H$ equivariant bounded derived category of $k$-sheaves where $H$ is either $B(\mathbb{C})$ or $I(\mathbb{C})$ depending on the setting (see [BL94] for more information about equivariant derived categories). In [JMW14a, §4.1] Juteau, Mautner and the second author introduce and prove the existence of parity sheaves on generalized flag varieties, a class of objects in $D_{H}^{b}(X)$ whose stalks satisfy a cohomological parity vanishing condition (for the trivial pariversity function). Their work was motivated by Soergel's idea to consider another class of objects as "replacements" for intersection cohomology complexes with positive characteristic coefficients (see [Soe00]). Observe that while $X$ is still a variety (resp. an indvariety) over $\mathbb{C}$ equipped with its classical topology, the coefficients of the sheaves we are studying may lie in a field of positive characteristic.

The theory of parity sheaves parallels the theory of perverse sheaves in the following points: 
(1) indecomposable parity sheaves are classified analogously to simple perverse sheaves, being up to isomorphism the unique extension of an irreducible local system on a stratum;

(2) in our setting, if the coefficients are a field of characteristic zero the intersection cohomology sheaves are parity sheaves.

But while the decomposition theorem for perverse sheaves fails in positive characteristic, the pushforward along a proper, even, stratified map preserves parity sheaves and it is possible to calculate the multiplicities of the occurring indecomposable parity sheaves via intersection forms (see [JMW14a, §3.3.]). Thus parity sheaves are particularly interesting in the case of positive characteristic coefficients.

Parity sheaves on various varieties have also been used for categorification. In [Mak15] Maksimau realizes Lusztig's integral form of the positive half of the quantum group associated to a Dynkin quiver as a coalgebra geometrically, by considering parity sheaves on quiver moduli spaces. In our setting, parity sheaves also give canonical bases of the Hecke algebra. If the coefficients are a field of characteristic zero, then the graded dimensions of stalks of parity sheaves give the Kazhdan-Lusztig basis (as mentioned above, see [KL80, Spr82]). For a field of positive characteristic the indecomposable parity sheaves realize the $p$-canonical basis in this way (see [WB12] in the setting of the flag variety) ${ }^{3}$.

From this perspective, analyzing when the $p$-canonical basis differs from the Kazhdan-Lusztig basis has several interesting geometric implications. First of all, it allows one to study the failure of the decomposition theorem in the modular setting (see [JMW14a, §3]). Secondly, there are close connections between the decomposition matrix for intersection cohomology complexes and the base change matrix between the Kazhdan-Lusztig and the $p$-canonical basis. In [AR14, Theorem 2.6] Achar and Riche show that the base change matrix gives a $q$-refinement of the decomposition matrix on the Langlands dual flag variety. Moreover, in [Wil14] the second author proves that certain base change coefficients and decomposition numbers coincide and uses this to give an example of a reducible characteristic variety in type $A$. Non-trivial decomposition numbers for an intersection cohomology complex can only occur when the characteristic variety of the corresponding regular holonomic $D_{X}$-module is reducible (see [VW13]).

\footnotetext{
${ }^{3}$ In many settings this statement follows from [Soe07, FW14, AR13]. In the generality discussed in this paper this result will appear in [RW15].
} 
1.3. This article gives a survey on known results about the $p$ canonical basis and its main purpose is to discuss an algorithm to calculate the $p$-canonical basis. This algorithm relies heavily on the diagrammatic description of the Hecke category $\mathbf{H}$. Motivated by de Cataldo and Migliorini's proof of the decomposition theorem (see [dCM09]), the second author showed in [JMW14a, §3.3] together with Juteau and Mautner that certain intersection forms govern the decomposition behaviour of the pushforward of the constant sheaf along a surjective, stratified, even resolution of singularities. In the diagrammatic framework this algorithm becomes more feasible, thanks to an explicit description of bases of Hom spaces. This leads to the algorithm described in this paper. We conclude the paper by giving many examples to show that the $p$-canonical basis may depend in a subtle way on $p$.

\subsection{Structure of the Paper:}

Section 2: We introduce notation and recall important results about the Hecke algebra and Soergel calculus.

Section 3: After recalling the definition of the $p$-canonical basis, we explain how to calculate it using intersection forms.

Section 4: The elementary properties of the $p$-canonical basis are stated and proved.

Section 5: We give several new and interesting examples of the p-canonical basis.

1.5. Acknowledgements. We would like to thank Ben Elias for very valuable comments and some minor corrections. We would also like to thank Nicolas Libedinsky for his feedback and the Max Planck Institute of Mathematics for financial support.

\section{Background}

2.1. Coxeter Systems and Based Root Data. Let $S$ be a finite set and $\left(m_{s, t}\right)_{s, t \in S}$ be a matrix with entries in $\mathbb{N} \cup\{\infty\}$ such that $m_{s, s}=1$ and $m_{s, t}=m_{t, s} \geqslant 2$ for all $s \neq t \in S$. Denote by $W$ the group generated by $S$ subject to the relations $(s t)^{m_{s, t}}=1$ for $s, t \in S$ with $m_{s, t}<\infty$. We say that $(W, S)$ is a Coxeter system and $W$ a Coxeter group. The Coxeter group $W$ comes equipped with a length function $l: W \rightarrow \mathbb{N}$ and the Bruhat order $\leqslant$ (see [Hum90] for more details). A Coxeter system $(W, S)$ is called crystallographic if $m_{s, t} \in\{2,3,4,6, \infty\}$ for all $s, t \in S$. 
Define an expression to be a finite sequence of elements in $S$. We denote by

$$
\operatorname{Ex}(S):=\{\varnothing\} \cup \bigcup_{i \in \mathbb{N} \backslash\{0\}} \underbrace{S \times \cdots \times S}_{i \text {-times }}
$$

the set of all expressions in $S$. For an expression $\underline{w}=\left(s_{1}, s_{2}, \ldots, s_{n}\right)$ denote its length by $l(\underline{w})=n$. The multiplication gives a canonical map $\operatorname{Ex}(S) \rightarrow W, \underline{w} \mapsto \underline{w}$. An expression $\underline{w}$ in $S$ is called reduced if $l(\underline{w})=$ $l(\underline{w}$.$) . For an expression \underline{w}=\left(s_{1}, s_{2}, \ldots, s_{n}\right)$ in $S$ a subexpression of $\underline{w}$ is a sequence $\underline{w}^{e}=\left(s_{1}^{e_{1}}, s_{2}^{e_{2}}, \ldots s_{n}^{e_{n}}\right)$ where $e_{i} \in\{0,1\}$ for all $i$. The sequence $\underline{e}=\left(e_{1}, e_{2}, \ldots, e_{k}\right)$ is called the associated 01-sequence. We usually decorate $\underline{e}$ as follows: For $1 \leqslant k \leqslant n$ denote by $\underline{w}_{\leqslant k}:=\left(s_{1}, s_{2}, \ldots, s_{k}\right)$ the first $k$ terms and set $w_{k}:=\left(\underline{w}_{\leqslant k}^{\underline{e}}\right)$. . Assign to $e_{i}$ a decoration $d_{i} \in\{U, D\}$ where $U$ stands for $U p$ and $D$ for Down as follows:

$$
d_{i}:= \begin{cases}U & \text { if } w_{i-1} s_{i}>w_{i-1}, \\ D & \text { if } w_{i-1} s_{i}<w_{i-1}\end{cases}
$$

We often write the decorated sequence as $\left(d_{1} e_{1}, d_{2} e_{2}, \ldots, d_{n} e_{n}\right)$. The defect of $\underline{e}$ is defined to be

$$
\operatorname{df}(e):=\left|\left\{i \mid d_{i} e_{i}=U 0\right\}\right|-\left|\left\{i \mid d_{i} e_{i}=D 0\right\}\right| .
$$

To illustrate the definitions, consider for example the case $S=\{s, t\}$ and $m_{s, t}=m_{t, s}=3$ (i.e. type $A_{2}$ ). The reduced expression $(s, t, s)$ admits two decorated 01-sequences expressing $s$ :

$$
\begin{array}{ll}
(U 1, U 0, D 0) & \text { of defect } 0 \\
(U 0, U 0, U 1) & \text { of defect } 2
\end{array}
$$

Recall that given an abstract root datum $\Psi=\left(X, \Phi, X^{\vee}, \Phi^{\vee}\right)$ and a basis $\Delta \subseteq \Phi$ the quadruple $\Psi_{0}=\left(X, \Delta, X^{\vee}, \Delta^{\vee}\right)$ is called a based root datum where $\Delta^{\vee}$ is the set of simple coroots (see [Spr89, §7.4] for the definition of a root datum). From now on, fix a based root datum $\Psi_{0}$. The matrix $\left(\left\langle\alpha_{s}, \alpha_{t}^{\vee}\right\rangle\right)_{s, t \in S}$ is called the Cartan matrix associated to this based root datum. By the existence theorem (see [SGA70, Exposé XXV, Théorème 1.1]), starting from $\Psi_{0}$ we get $G$, a split connected reductive algebraic group scheme over $\mathbb{Z}$, together with a Borel subgroup $B \subseteq G$ and a maximal torus $T \subseteq B \subseteq G$ such that the root datum determined by $(G, T)$ and the basis given by the simple roots whose root groups are contained in $B$ give the corresponding based root datum $\Psi_{0}$. For a root $\alpha \in \Phi$ we define the corresponding reflection on $X$ via

$$
s_{\alpha} \lambda:=\lambda-\left\langle\lambda, \alpha^{\vee}\right\rangle \alpha \quad \text { for all } \lambda \in X
$$


We denote the Weyl group of $\Phi$ by $W:=\left\langle s_{\alpha} \mid \alpha \in \Phi\right\rangle$.

For $S:=\left\{s_{\alpha} \mid \alpha \in \Delta\right\}$ the triple $\left(X^{\vee}, \Delta, \Delta^{\vee}\right)$ gives a (not necessarily symmetric) faithful realization of the Coxeter system $(W, S)$ over $\mathbb{Z}$ (as defined in the appendix of [Eli13]). Fix a commutative ring $k$. Then ${ }^{k} V:=X^{\vee} \otimes_{\mathbb{Z}} k$ yields a (potentially non-faithful) realization of $(W, S)$ over $k$. Set ${ }^{k} V^{*}:=\operatorname{Hom}_{k}\left({ }^{k} V, k\right)$ and note that ${ }^{k} V^{*}$ is isomorphic to $X \otimes_{\mathbb{Z}} k$. Throughout we will assume the following:

Assumption 2.1 (Demazure Surjectivity). The maps $\alpha_{s}:{ }^{k} V \rightarrow k$ and $\alpha_{s}^{\vee}:{ }^{k} V^{*} \rightarrow k$ are surjective for all $s \in S$.

This is automatically satisfied if 2 is invertible in $k$ or if the Coxeter system $(W, S)$ is of simply-laced type and of rank $|S| \geqslant 2$.

We denote by $R=S\left({ }^{k} V^{*}\right)$ the symmetric algebra of ${ }^{k} V^{*}$ over $k$ and view it as a graded ring with ${ }^{k} V^{*}$ in degree 2 . The action of $W$ on ${ }^{k} V$ induces an action on $R$ by functoriality. For any $s \in S$ we define the Demazure operator $\partial_{s}: R \rightarrow R(-2)$ via

$$
\partial_{s}(f):=\frac{f-s(f)}{\alpha_{s}}
$$

where (1) denotes the grading shift down by one: Given a graded $R$ bimodule $B=\bigoplus_{i \in \mathbb{Z}} B^{i}$, we denote by $B(1)$ the shifted bimodule with $B(1)^{i}=B^{i+1}$. Observe that $\partial_{s}$ is a well-defined graded $R^{s}$-bimodule homomorphism (see [EW13, §3.3] for more details).

2.2. The Hecke Algebra. The Hecke algebra is the free $\mathbb{Z}\left[v, v^{-1}\right]$ algebra with $\left\{H_{w} \mid w \in W\right\}$ as basis and multiplication determined by:

$$
\begin{aligned}
H_{s}^{2} & =\left(v^{-1}-v\right) H_{s}+1 & & \text { for all } s \in S, \\
H_{x} H_{y} & =H_{x y} & & \text { if } l(x)+l(y)=l(x y) .
\end{aligned}
$$

There is a unique $\mathbb{Z}$-linear involution $\overline{(-)}$ on $\mathcal{H}$ satisfying $\bar{v}=v^{-1}$ and $\overline{H_{x}}=H_{x^{-1}}^{-1}$. The Kazhdan-Lusztig basis element $\underline{H}_{x}$ is the unique element in $H_{x}+\sum_{y<x} v \mathbb{Z}[v] H_{y}$ that is invariant under $\overline{(-)}$. This is Soergel's normalization from [Soe97] of a basis introduced originally in [KL79].

2.3. Soergel Calculus. We define an $S$-graph to be a finite, decorated, planar graph with boundary properly embedded in the planar strip $\mathbb{R} \times[0,1]$ whose edges are coloured by $S$ and all of whose vertices are of the following types: 


$$
\begin{array}{ll} 
& \\
&
\end{array}
$$

where we require the $2 m_{s, t}$-valent vertex to have exactly $2 m_{s, t}$ edges, coloured alternately by $s$ and $t$ around the vertex.

The regions of an $S$-graph (i.e. the connected components of the complement of the graph in $\mathbb{R} \times[0,1])$ may be decorated by homogeneous elements of $R$. The degree of a decorated $S$-graph is defined as the sum of the degrees of its vertices and of the degrees of the polynomials decorating its regions.

Next, we introduce the diagrammatic category of Soergel bimodules. The main reference for this is [EW13] (see also [Eli13] in the dihedral case and [EK10] in type $A$ ).

Let $\mathbf{H}_{B S}$ be the strict monoidal category with $\mathbb{Z}$-graded Hom-spaces which is monoidally generated by the elements in $S$. Thus the objects of $\mathbf{H}_{B S}$ are given by $\operatorname{Ex}(S)$ and the monoidal structure on the level of objects is given by concatenation of sequences in $S$. For any $\underline{x}, y \in$ $\operatorname{Ex}(S), \operatorname{Hom}_{\mathbf{H}_{B S}}(\underline{x}, \underline{y})$ is defined to be the free $R$-module generated by isotopy classes of decorated $S$-graphs with bottom boundary $\underline{x}$ and top boundary $y$ modulo the local relations below. The composition (resp. tensor product) of two morphisms is given by vertical (resp. horizontal) concatenation of diagrams.

We now recall the relations defining $\mathbf{H}_{B S}$ :

2.3.1. One-colour Relations. For all $s \in S$ we have:

- Frobenius Unit:

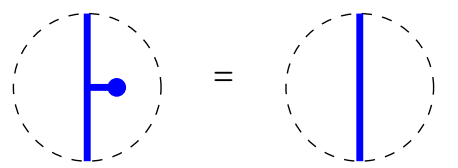

- Frobenius Associativity:

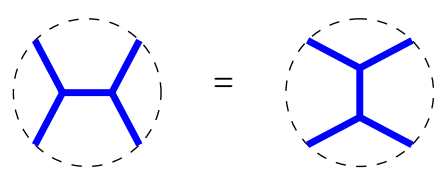


- Needle Relation:

$$
\mathrm{O}_{i}^{\prime} \mathrm{O}_{i}=0
$$

- Barbell Relation:

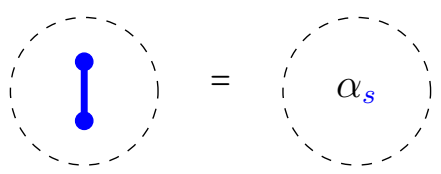

- Nil Hecke Relation:

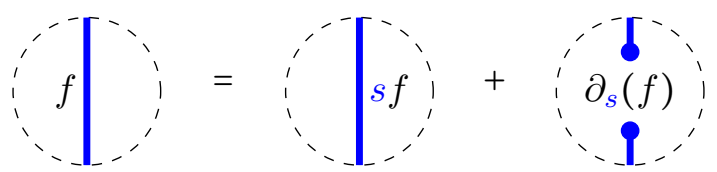

2.3.2. Two-colour Relations. There are two colour relations for all pairs $s, t \in S$ such that $m_{s, t}<\infty$ (so that the $2 m_{s t}$-valent vertex is defined).

The first two-colour relation is called Two-colour Associativity and describes what happens what happens when we pull a trivalent vertex through a $2 m_{s, t}$-valent vertex. We give it for $m_{s, t} \in\{2,3,4\}$ and let the reader guess the general form (see [Eli13, (6.12)]):

$$
\text { if } m_{s, t}=2 \quad\left(\text { type } A_{1} \times A_{1}\right)
$$

The next two-colour relation is called Jones-Wenzl Relation and

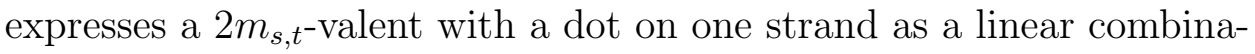
tion of diagrams in which only dots and trivalent vertices appear. We state it only for $m_{s, t} \in\{2,3,4\}$ and refer the reader to [Eli13] for more detail:

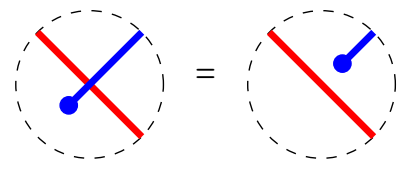

$$
\text { if } m_{s, t}=2 \text {, }
$$




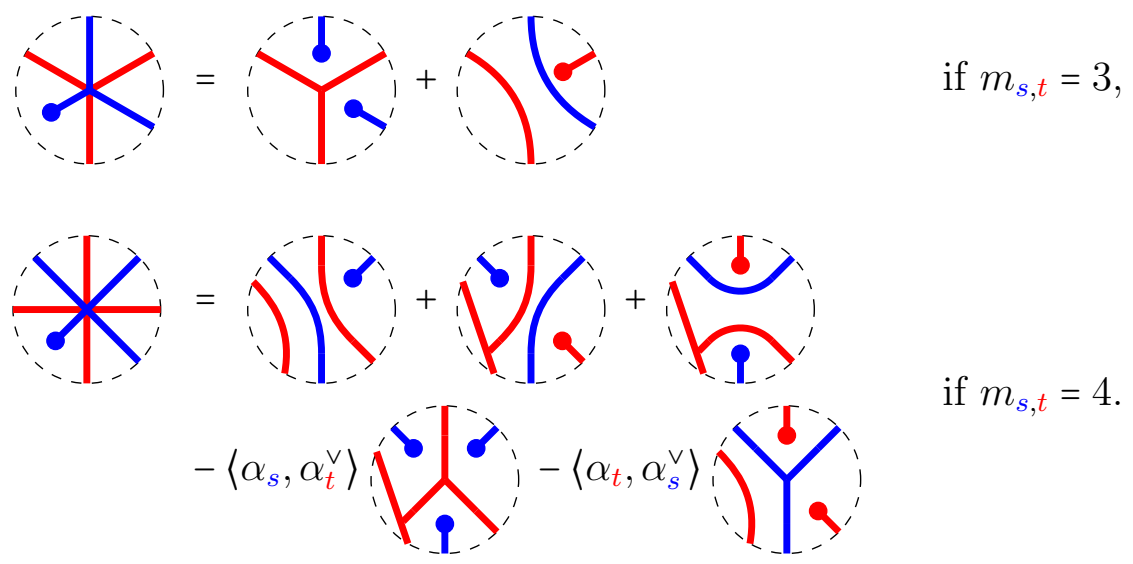

2.3.3. Three-Colour Relations. We do not repeat the definition of the Zamolodchikov relations or "higher braid relations" here. The reader can find them in $[\mathbf{E W 1 3}, \S 1.4 .3]$ and is referred to [EW12] for more detail on the topological origins of the Zamolodchikov relations.

2.4. Light Leaves and Double Leaves. In this section we briefly discuss how to describe bases for morphism spaces in $\mathbf{H}_{B S}$. Fix an expression $\underline{w}$ and a reduced expression $\underline{x}$. In $[\mathbf{E W} 13, \S 6.1]$ it is described how one may associate a "light leaves morphism" $\mathrm{LL}_{\underline{w}, \underline{e}} \epsilon$ $\operatorname{Hom}_{\mathbf{H}_{B S}}(\underline{w}, \underline{x})$ to each subexpression $\underline{e}$ of $\underline{w}$ such that $(\underline{w} \underline{e}) \bullet=x$. We will not need the explicit construction here, but the reader is encouraged to consult [EW13, §6.1] to follow our examples. The construction of light leaves follows a construction of Libedinsky for Soergel bimodules [Lib08] and depends on certain additional non-canonical choices.

In the special case of $x=e$, the identity of $W$, we get (see [EW13, Proposition 6.12]):

Proposition 2.2. The set of all light leaves indexed by subsequences $\underline{e}$ of $\underline{w}$ expressing the identity of $W$ gives an $R$-basis of $\operatorname{Hom}_{\mathbf{H}_{B S}}(\underline{w}, \varnothing)$.

For an $S$-graph $D$ denote by $\bar{D}$ the $S$-graph obtained by flipping the diagram upside down. This induces a contravariant equivalence on the monoidal category $\mathbf{H}_{B S}$ fixing all objects.

Out of light leaves one can construct double leaves as follows. Let $\underline{x}$ and $\underline{y}$ be arbitrary expressions in $S$. For any subsequences $\underline{e}$ (resp. $\underline{f}$ ) of $\underline{x}$ (resp. $\underline{y}$ ) both expressing $w \in W$ define $\mathbb{L}_{w, \underline{e}, \underline{f}}:=\overline{\mathrm{LL}_{\underline{y}, f}} \circ \mathrm{LL}_{\underline{x}, \underline{e}}$. The following result can be found in [EW13, Theorem 6.11] (and was proved earlier in the setting of Soergel bimodules by Libedinsky in [Lib15]): 
Theorem 2.3. The set of all double leaves ranging over all $w \in W$ and pairs of subsequences $\underline{e}$ (resp. $\underline{f}$ ) of $\underline{x}$ (resp. $\underline{y}$ ) both expressing w gives an $R$-basis of $\operatorname{Hom}_{\mathbf{H}_{B S}}(\underline{x}, \underline{y})$.

2.5. The Diagrammatic Category: Properties. Note that all relations in $\mathbf{H}_{B S}$ are homogeneous for our grading on $S$-graphs and thus $\mathbf{H}_{B S}$ is a category enriched in graded left $R$-modules; multiplying an $S$-graph $D$ with a homogeneous polynomial $f \in R$ from the left is defined by decorating the leftmost region of $D$ with $f$.

Let $\mathbf{H}$ be the Karoubian envelope of the graded version of the additive closure of $\mathbf{H}_{B S}$, in symbols $\mathbf{H}=\mathcal{K} \operatorname{ar}\left(\mathbf{H}_{B S}\right)$. We call $\mathbf{H}$ the diagrammatic category of Soergel bimodules. In other words, in the passage from $\mathbf{H}_{B S}$ to $\mathbf{H}$ we first allow direct sums and grading shifts (restricting to degree preserving homomorphisms) and then the taking of direct summands. The following properties can be found in [EW13, Lemma 6.24, Theorem 6.25 and Corollary 6.26]:

Theorem 2.4 (Properties of $\mathbf{H}$ ).

Let $k$ be a complete local ring (e.g. a field or the p-adic integers $\mathbb{Z}_{p}$ ).

(1) $\mathbf{H}$ is a Krull-Schmidt category.

(2) For all $w \in W$ there exists a unique, indecomposable object $B_{w} \in \mathbf{H}$ which is a direct summand of $\underline{w}$ for any reduced expression $\underline{w}$ of $w$ and which is not isomorphic to a grading shift of any direct summand of any expression $\underline{v}$ for $v<w$. The object $B_{w}$ does not depend up to isomorphism on the reduced expression $\underline{w}$ of $w$.

(3) The set $\left\{B_{w} \mid w \in W\right\}$ gives a complete set of representatives of the isomorphism classes of indecomposable objects in $\mathbf{H}$ up to grading shift.

(4) There exists a unique isomorphism of $\mathbb{Z}\left[v, v^{-1}\right]$-algebras

$$
\operatorname{ch}:[\mathbf{H}] \longrightarrow \mathcal{H}
$$

sending $\left[B_{s}\right]$ to $\underline{H}_{s}$ for all $s \in S$, where $[\mathbf{H}]$ denotes the split Grothendieck group of $\mathbf{H}$. (We view $[\mathbf{H}]$ as a $\mathbb{Z}\left[v, v^{-1}\right]$-algebra as follows: the monoidal structure on $\mathbf{H}$ induces a unital, associative multiplication and $v$ acts via $v[B]:=[B(1)]$ for an object $B$ of $\mathbf{H}$.)

It should be noted that we do not have a diagrammatic presentation of $\mathbf{H}$ as determining the idempotents in $\mathbf{H}_{B S}$ is usually extremely difficult. 
Observe that $\overline{(-)}$ extends to a contravariant equivalence of the graded, $R$-linear, additive, monoidal category $\mathbf{H}$ sending $B_{w}(n)$ to $B_{w}(-n)$ for all $n \in \mathbb{Z}$ and $w \in W$.

In order to explicitly give the isomorphism in the last part of Theorem 2.4, we need to introduce some more notation. For $x \in W$, let $\mathbf{H}^{\nless x}$ be the quotient category of $\mathbf{H}$ by the 2-sided ideal of morphisms factoring through any grading shift of a reduced expression $y$ for some $y<x$. Write $\operatorname{Hom}_{\nless x}(-,-)$ for homomorphism spaces in $\mathbf{H}^{\not x}$. In $\mathbf{H}^{\nless x}$ any two reduced expressions for $x$ become canonically isomorphic. We denote the image of any reduced expression for $x$ in $\mathbf{H}^{\nless x}$ by $x$ as well. Under the assumptions of Theorem 2.4 we can define the diagrammatic character on an object $B$ of $\mathbf{H}$ as follows:

$$
\begin{aligned}
\operatorname{ch}:[\mathbf{H}] & \longrightarrow \mathcal{H}, \\
{[B] } & \longmapsto \sum_{w \in W} \operatorname{grk} \operatorname{Hom}_{\nless w}^{\bullet}(B, w) H_{w}
\end{aligned}
$$

and extend $\mathbb{Z}\left[v, v^{-1}\right]$-linearly. In the last definition grk denotes the graded rank of the free $R$-module of homomorphisms of all degrees

$$
\operatorname{Hom}_{\nless w}^{\bullet}\left(B_{x}, w\right):=\bigoplus_{n \in \mathbb{Z}} \operatorname{Hom}_{\nless w}\left(B_{x}, w(n)\right) .
$$

\section{The $p$-Canonical Basis and Intersection Forms}

In this section we recall the definition of the $p$-canonical basis of the Hecke algebra (see [Wil12]) and explain how to calculate it using intersection forms. Fix a field $k$ of characteristic $p \geqslant 0$ and the realization ${ }^{k} V$ of $(W, S)$. We use this realization to define $\mathbf{H}$.

It is an interesting question what basis of the Hecke algebra the classes of the self-dual indecomposable objects in $\mathbf{H}$ correspond to. The answer is given for $k=\mathbb{R}$ by Soergel's conjecture which Elias and the second author proved in [EW14].

Theorem 3.1 (Elias-W. 2013). $\operatorname{ch}\left(\left[B_{w}\right]\right)=\underline{H}_{w}$ for all $w \in W$.

This illustrates that the basis of self-dual indecomposable objects in $\mathbf{H}$ gives an extremely interesting basis of $\mathcal{H}$ for $k=\mathbb{R}$ and motivates our definition of the $p$-canonical basis.

Definition 3.2. Define ${ }^{p} \underline{H}_{w}=\operatorname{ch}\left(\left[B_{w}\right]\right)$ for all $w \in W$ where ch : $[\mathbf{H}] \stackrel{\cong}{\longrightarrow} \mathcal{H}$ is the isomorphism of $\mathbb{Z}\left[v, v^{-1}\right]$-algebras introduced earlier, and $p$ denotes the characteristic of $k$ as above.

Theorem 2.4 implies that $\left\{{ }^{p} \underline{H}_{w} \mid w \in W\right\}$ gives a basis of $\mathcal{H}$, called the $p$-canonical basis. As will become clearer later, the $p$-canonical 
basis depends only on the type of the root system chosen and on the characteristic $p$ of the field $k$.

Next, we are going to explain how to use intersection forms to explicitly calculate the $p$-canonical basis. In order to calculate ${ }^{p} \underline{H}_{w}$ we proceed by induction on $l(w)$. The induction start is given by ${ }^{p} \underline{H}_{e}=\underline{H}_{e}=H_{e}$. Assume that we have already calculated ${ }^{p} \underline{H}_{v}$ for all $v<w$. Let $\underline{w}$ be an arbitrary reduced expression for $w$. According to Theorem 2.4, we need to decompose $\underline{w}$ into indecomposable objects $B_{x}(n)$ for $x \in W$ and $n \in \mathbb{Z}$ in $\mathbf{H}$. For this we need local intersection forms:

Write $\operatorname{Hom}_{\nless x, k}(-,-)$ for the homomorphism spaces in $k \otimes_{R} \mathbf{H}^{\nless x}$ where we kill the action of the unique maximal ideal of all polynomials of positive degree. Since $\operatorname{Hom}_{\nless x}^{\bullet}(\underline{w}, x)$ is a graded free $R$-module with basis (see Section 2.4):

$$
\left\{\mathrm{LL}_{\underline{w}, \underline{e}} \mid \underline{e} \text { is a subexpression of } \underline{w} \operatorname{expressing} x\right\},
$$

$\operatorname{Hom}_{\nless x, k}^{\bullet}(\underline{w}, x)$ is a graded $k$-vector space on the same basis.

For an arbitrary expression $\underline{w}$ in $S$ and $x \in W$, consider the $k$ bilinear Hom-pairing

$$
\begin{aligned}
\operatorname{Hom}_{\nless x, k}^{\bullet}(x, \underline{w}) \times \operatorname{Hom}_{\nless x, k}^{\bullet}(\underline{w}, x) & \longrightarrow \operatorname{End}_{\nless x, k}^{\bullet}(x)=k, \\
(f, g) & \longmapsto g \circ f .
\end{aligned}
$$

Observe that $\operatorname{End}_{\nless x, k}^{\bullet}(x)$ is concentrated in degree 0 and that the duality $\overline{(-)}$ on $\mathbf{H}$ gives an isomorphism between $\operatorname{Hom}_{\nless x, k}^{\bullet}(x, \underline{w})$ and $\overline{\operatorname{Hom}_{\nless x, k}^{\bullet}(\underline{w}, x)}$. This allows us to define:

Definition 3.3. The local intersection form of $\underline{w}$ at $x$ is the $k$ bilinear pairing on the graded free $k$-vector space $\operatorname{Hom}_{\nless x, k}^{\bullet}(\underline{w}, x)$ given by

$$
\begin{aligned}
I_{\underline{w}, x}: \operatorname{Hom}_{\nless x, k}^{\bullet}(\underline{w}, x) \times \operatorname{Hom}_{\nless x, k}^{\bullet}(\underline{w}, x) & \longrightarrow \operatorname{End}_{\nless x, k}(x)=k, \\
(f, g) & \longmapsto g \circ \bar{f} .
\end{aligned}
$$

The local intersection form of $\underline{w}$ at $x$ can be split up into degree pieces as follows: Since $\operatorname{End}_{\nless x, k}^{\bullet}(x)$ is concentrated in degree 0, a homomorphism in $\operatorname{Hom}_{\nless x, k}^{\bullet}(\underline{w}, x(d))$ for some $d \in \mathbb{Z}$ can only pair nontrivially with elements of $\operatorname{Hom}_{\nless x, k}^{\bullet}(\underline{w}, x(-d))$. The $d$-th grading piece of the intersection form can thus be defined as:

$$
I_{\underline{w}, x}^{d}: \operatorname{Hom}_{\nless x, k}(\underline{w}, x(-d)) \times \operatorname{Hom}_{\nless x, k}(\underline{w}, x(d)) \longrightarrow \operatorname{End}_{\nless x, k}(x(d))=k
$$


Finally, the graded rank of $I_{\underline{w}, x}$ is denoted by $n_{x, w} \in \mathbb{Z}_{\geqslant 0}\left[v, v^{-1}\right]$ and defined as

$$
n_{x, w}:=\sum_{d \in \mathbb{Z}} \operatorname{rk}\left(I_{\underline{w}, x}^{d}\right) v^{d} .
$$

The following lemma illustrates the importance of intersection forms for the calculation of the $p$-canonical basis and follows from an argument similar to [JMW14a, Lemma 3.1]:

Lemma 3.4. The multiplicity of $B_{x}$ in $\underline{w}$ in $\mathbf{H}$ is given by the graded rank of $I_{\underline{w}, x}$.

After calculating the graded ranks of all $I_{\underline{w}, x}$ for $x<w$, we can write for $\underline{w}=s_{1} s_{2} \ldots s_{n}$ :

$$
\underline{H}_{s_{1}} \underline{H}_{s_{2}} \cdots \underline{H}_{s_{n}}={ }^{p} \underline{H}_{w}+\sum_{x<w} n_{x, w}^{p} \underline{H}_{x} .
$$

REMARK 3.5. By comparing the intersection forms over $\mathbb{Q}$ and $k$, one may deduce that one only needs to calculate the graded ranks of $I_{\underline{w}, x}$ for those $x$ such that $\underline{H}_{x}$ occurs with a non-trivial coefficient when expressing $\underline{H}_{s_{1}} \underline{H}_{s_{2}} \ldots \underline{H}_{s_{n}}$ in terms of the Kazhdan-Lusztig basis.

In order to determine ${ }^{p} \underline{H}_{w}$ we have to invert the matrix $\left(n_{x, y}\right)_{x, y \leqslant w}$ which is upper triangular with ones on the diagonal in any total order refining the Bruhat order.

Finally, it should be noted that in practice one calculates the intersection form once over $\mathbb{Z}$ and reduces modulo different primes.

REMARK 3.6. There are other ways to calculate the $p$-canonical basis (which, however, are much more difficult in practice).

(1) If one can describe the geometry of the corresponding Schubert varieties quite explicitly, one can do calculations using parity sheaves (see [WB12, appendix]).

(2) In [FW14] Fiebig and the second author show that for a field $k$ of characteristic $p$ (or more generally a complete local PID), the Braden-MacPherson algorithm on the Bruhat graph allows one to compute the $p$-canonical basis.

3.1. Calculations in the nil Hecke Ring. In [HW15] Xuhua $\mathrm{He}$ and the second author reduce the calculation of certain entries in the intersection form to a simple formula in the nil Hecke ring. Instead of going into too much detail, we will try to give a survey of these results.

First, recall the definition of the nil Hecke ring. Let $Q$ be the field of fractions of $R$. Denote by $Q * W$ the smash product. In other words, $Q * W$ is the free left $Q$-module with basis $\left\{\delta_{w} \mid w \in W\right\}$ and 
multiplication given by

$$
\left(f \delta_{x}\right)\left(g \delta_{y}\right)=f(x g) \delta_{x y}
$$

for $f, g \in Q$ and $x, y \in W$. Inside $Q * W$, we consider the elements

$$
D_{s}=\frac{1}{\alpha_{s}}\left(\delta_{e}-\delta_{s}\right)=\left(\delta_{e}+\delta_{s}\right) \frac{1}{\alpha_{s}}
$$

which satisfy the following relations:

$$
\begin{aligned}
D_{s}^{2} & =0, \\
D_{s} f & =(s f) D_{s}+\partial_{s}(f) \text { for all } f \in Q, \\
\underbrace{D_{s} D_{t} D_{s} \ldots}_{m_{s, t} \text { terms }} & =\underbrace{D_{t} D_{s} D_{t} \ldots}_{m_{s, t} \text { terms }} .
\end{aligned}
$$

The last relation ensures that for $y \in W$ and any reduced expression $\underline{y}=\left(s_{1}, s_{2}, \ldots, s_{n}\right)$ of $y$ we get well-defined elements

$$
D_{y}=D_{s_{1}} D_{s_{2}} \ldots D_{s_{n}} \in Q * W .
$$

The nil Hecke ring $\mathcal{N H}$ is the left $R$-submodule of $Q * W$ generated by $\left\{D_{y} \mid v \in W\right\}$.

Next, we briefly introduce gobbling morphisms. For any expression $\underline{w}=\left(s_{1}, s_{2}, \ldots, s_{n}\right)$ in $S$, consider the following 01-sequence $\underline{e}$ with:

$$
e_{i}= \begin{cases}1 & \text { if } w_{i-1} s_{i}>w_{i-1}, \\ 0 & \text { otherwise }\end{cases}
$$

where at each step $w_{i}$ is defined as in Section 2.1. Note that $\left(\underline{w}^{\underline{e}}\right)$. is the maximal element in $W$ expressible as a subexpression of $\underline{w}$, and that the decoration of $\underline{e}$ consists entirely of $U 1$ 's and $D 0$ 's. Therefore any choice of light leaf morphism $L_{\underline{w}, \underline{e}}$ has degree $l((\underline{w} \underline{e}) \bullet)-l(\underline{w})$ and consists only of $2 m_{s t}$-valent and trivalent vertices. Denote by $G_{\underline{w}}$ the image of $\mathrm{LL}_{\underline{w}, \underline{e}}$ in $\mathbf{H}^{\nless\left(\underline{w}^{e}\right)} \cdot$. The morphism $G_{\underline{w}}$ is called a gobbling morphism and can be characterized as follows (see [HW15, Proposition 3.4]):

Proposition 3.7. Let $\underline{w}$, $\underline{e}$ be as above. Any morphism $\underline{w} \rightarrow(\underline{w}-\underline{e})$. in $\mathbf{H}^{\nless\left(\underline{w}^{e}\right)}$ • given by diagrams consisting only of $2 m_{\text {st }}$-valent vertices and $l(\underline{w})-l((\underline{w} \underline{e})$.$) trivalent vertices is equal to G_{\underline{w}}$.

From this they deduce the canonicity of any light leaf morphism $\mathrm{LL}_{\underline{w}, f}$ in $\mathbf{H}^{\nless\left(\underline{w}^{\underline{f}}\right)}$ • indexed by a 01-sequence $\underline{f}$ without $D 1$ 's in its decoration. (This follows because the morphism is given as the composition of a sequence of dots on strands corresponding to $U 0$ 's followed by a gobbling morphism.)

Finally, we come to their formula in the nil Hecke ring for certain entries of the intersection form. Let $\underline{e^{1}}$ and $\underline{e^{2}}$ be two subexpressions 
of $\underline{w}$. Assume that $\frac{e^{1}}{2}$ and $\underline{e^{2}}$ both express the same element $x \in W$ (i.e. $x=\left(\underline{w} \underline{e^{1}}\right) \bullet=\left(\underline{w} \underline{e^{2}}\right)$.) and that their decorations do not contain any $D 1$. Define an element of the nil Hecke ring as the product $f\left(\underline{e^{1}}, \underline{e^{2}}\right)=$ $f_{1} f_{2} \ldots f_{m}$ where

$$
f_{i}= \begin{cases}\alpha_{s_{i}} & \text { if } e_{i}^{1}=e_{i}^{2}=U 0, \\ 1 & \text { if exactly one of } e_{i}^{1} \text { and } e_{i}^{2} \text { is } U 0, \\ D_{s_{i}} & \text { otherwise. }\end{cases}
$$

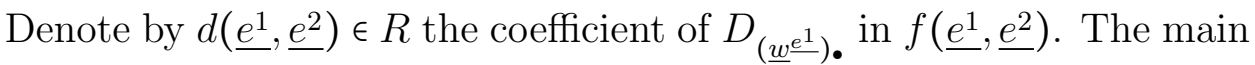
result is [HW15, Theorem 5.1]:

Theorem 3.8. For $\underline{e^{1}}$ and $\underline{e^{2}}$ as above, we have

$$
I_{\underline{w}, x}\left(\mathrm{LL}_{\underline{w}, \underline{e^{1}}}, \mathrm{LL}_{\underline{w}, \underline{e^{2}}}\right)=d\left(\underline{e^{1}}, \underline{e^{2}}\right) .
$$

This theorem gives a combinatorial formula for some entries in the intersection form. Sometimes one is lucky, and it can be used to calculate the complete intersection form, as we will see in examples below.

\section{First Properties of the $p$-Canonical Basis}

The goal of this section is to prove elementary properties of the $p$-canonical basis and to compare it to the Kazhdan-Lusztig basis. For this we need a $p$-modular system. Let $\mathbb{O}$ be a complete local ring with residue field $k$ of characteristic $p>0$ and quotient field $\mathbb{K}$ of characteristic 0 . Fix the realization ${ }^{\mathbb{O}} V$ of $(W, S)$ and use it to define $\mathbf{H}$. For $x \in W$ we will denote by $B_{x}$ (resp. ${ }^{k} B_{x}$ or ${ }^{\mathbb{K}} B_{x}$ ) the indecomposable object in $\mathbf{H}$ (resp. ${ }^{k} \mathbf{H}:=\mathbf{H} \otimes_{\mathbb{O}} k$ or ${ }^{\mathbb{K}} \mathbf{H}:=\mathbf{H} \otimes_{\mathbb{O}} \mathbb{K}$ ).

The following lemma shows that indecomposable objects in $\mathbf{H}$ remain indecomposable when passing to ${ }^{k} \mathbf{H}$.

Lemma 4.1. We have for all $w \in W: B_{w} \otimes_{\mathbb{O}} k \cong{ }^{k} B_{w}$.

Proof. Assume $B_{w} \otimes_{\mathbb{O}} k$ is not indecomposable in ${ }^{k} \mathbf{H}$. Then there exists a non-trivial idempotent $e \in \operatorname{End}_{k_{\mathbf{H}}}\left(B_{w} \otimes_{\mathbb{O}} k\right)$. Since $\operatorname{End}_{\mathbf{H}}\left(B_{w}\right)$ is a finitely generated $\mathbb{O}$-module, we can use idempotent lifting techniques for complete local rings (see [Lam01, Theorem 21.31]) and find an idempotent $\tilde{e} \in \operatorname{End}_{\mathbf{H}}\left(B_{w}\right)$ mapping to $e$ in $\operatorname{End}_{k_{\mathbf{H}}}\left(B_{w} \otimes_{\mathbb{O}} k\right) \cong$ $\operatorname{End}_{\mathbf{H}}\left(B_{w}\right) \otimes_{\mathbb{O}} k$. Since $B_{w}$ is indecomposable, this idempotent has to be trivial, a contradiction.

Some of the following properties can also be found in [WB12] and [Wil14]:

Proposition 4.2. For all $x, y \in W$ we have: 
(1) ${ }^{p} \underline{H}_{x}={ }^{p} \underline{H}_{x}$, i.e. ${ }^{p} \underline{H}_{x}$ is self-dual,

(2) ${ }^{p} \underline{H}_{x}=H_{x}+\sum_{y<x}{ }^{p} h_{y, x} H_{y}$ with ${ }^{p} h_{y, x} \in \mathbb{Z}_{\geqslant 0}\left[v, v^{-1}\right]$,

(3) ${ }^{p} \underline{\underline{H}}_{x}=\underline{H}_{x}+\sum_{y<x}{ }^{p} m_{y, x} \underline{H}_{y}$ with self-dual ${ }^{p} m_{y, x} \in \mathbb{Z}_{\geqslant 0}\left[v, v^{-1}\right]$,

(4) ${ }^{p} m_{y, x}=p_{y^{-1}, x^{-1}}$ for all $x, y \in W$,

(5) ${ }^{p} m_{y, x}=0$ unless $\mathcal{L}(x) \subseteq \mathcal{L}(y)$ and $\mathcal{R}(x) \subseteq \mathcal{R}(y)$ where $\mathcal{L}$ and $\mathcal{R}$ denote the left and right descent sets,

(6) ${ }^{p} \underline{H}_{x}{ }^{p} \underline{H}_{y}=\sum_{z \in W}{ }^{p} \mu_{x, y}^{z} \underline{H}_{z}$ with self-dual ${ }^{p} \mu_{x, y}^{z} \in \mathbb{Z}_{\geqslant 0}\left[v, v^{-1}\right]$,

(7) ${ }^{p} \underline{H}_{x}=\underline{H}_{x}$ for $p \gg 0$ (i.e. there are only finitely many primes for which ${ }^{p} \underline{H}_{x} \neq \underline{H}_{x}$ ).

Proof. (1) We proceed by induction on $l(x)$. For small $x$ the statement is clear as ${ }^{p} \underline{H}_{e}=\underline{H}_{e}$ and ${ }^{p} \underline{H}_{s}=\underline{H}_{s}$ for all $s \in S$ and all primes $p$. Assume that we have shown that ${ }^{p} \underline{H}_{y}$ is self-dual for $y<x$. Choose $s \in \mathcal{L}(x)$ and set $y=s x$. The characterization of ${ }^{k} B_{x}$ in Theorem 2.4 implies that ${ }^{k} B_{x}$ occurs with multiplicity one in ${ }^{k} B_{s}{ }^{k} B_{y}$ and that $\overline{{ }^{k} B_{x}(n)}={ }^{k} B_{x}(-n)$. Thus we can write

$$
{ }^{k} B_{s}{ }^{k} B_{y}={ }^{k} B_{x} \oplus \bigoplus_{\substack{z<x \\ n \in \mathbb{Z}}}\left({ }^{k} B_{z}(n)\right)^{\oplus a_{z, n}}
$$

with $a_{y, n} \in \mathbb{Z}_{\geqslant 0}$ for all $y<x$ and $n \in \mathbb{Z}$ and all but finitely many of the $a_{y, n}$ are zero. Applying the duality $\overline{(-)}$ to both sides and using that the left hand side is self-dual yields $a_{z, n}=a_{z,-n}$ for all $z<x$ and $n \in \mathbb{Z}$. This implies

$$
{ }^{p} \underline{H}_{S}^{p} \underline{H}_{y}={ }^{p} \underline{H}_{x}+\sum_{z<x}{ }^{p} \mu_{z, y}^{p} \underline{H}_{z}
$$

where ${ }^{p} \mu_{z, y}=\sum_{n \in Z} a_{z, n} v^{n} \in \mathbb{Z}_{\geqslant 0}\left[v, v^{-1}\right]$ is self-dual for all $z<$ $x$. Therefore the self-duality of the left-hand side and our induction hypothesis imply the self-duality of ${ }^{p} \underline{H}_{x}$.

(2) The unicity in the characterization of ${ }^{k} B_{x}$ in Theorem 2.4 implies that it occurs with multiplicity 1 in any reduced expression $\underline{x}$ of $x$. In the quotient $\mathbf{H}^{\nless x}$ all other direct summands of $x$ are killed. Thus we get grk $\operatorname{Hom}_{\nless x}\left(B_{x}, x\right)=1$. Note that the Laurent polynomials ${ }^{p} h_{y, x}$ have non-negative coefficients as they are given by graded ranks of free $R$-modules.

(3) According to (2), $\underline{H}_{x}$ occurs precisely with coefficient 1 in ${ }^{p} \underline{H}_{x}$. The self-duality of the Laurent polynomials ${ }^{p} m_{y, x}$ follows from (1) and the self-duality of the Kazhdan-Lusztig basis. Since $\operatorname{Hom}_{\nless w}^{\bullet}\left(B_{x}, w\right)$ is a free $R$-module, we get for $F \in\{k, \mathbb{K}\}$ :

$\operatorname{Hom}_{\nless w}^{\bullet}\left(B_{x}, w\right) \otimes_{\mathbb{O}} F \cong \operatorname{Hom}_{\mathbf{H}^{\nless w} \otimes_{\mathbb{O}} F}^{\cdot}\left(B_{x} \otimes_{\mathbb{O}} F, w \otimes_{\mathbb{O}} F\right)$. 
This implies in particular using Lemma 4.1:

$$
\operatorname{ch}\left({ }^{k} B_{x}\right)=\operatorname{ch}\left(B_{x}\right)=\operatorname{ch}\left(B_{x} \otimes_{\mathbb{O}} \mathbb{K}\right) .
$$

Thus the ${ }^{p} m_{y, x}$ have non-negative coefficients as they come from decomposing $B_{x} \otimes_{\mathbb{O}} \mathbb{K}$ into indecomposable objects of the form ${ }^{\mathbb{K}} B_{x}$ in ${ }^{\mathbb{K}} \mathbf{H}$ whose character is given by $\underline{H}_{x}$ by Theorem 3.1.

(4) There is an equivalence $\iota$ on ${ }^{k} \mathbf{H}$ viewed as a $k$-linear category induced by the horizontal flip of Soergel graphs. It maps $\left(s_{1}, s_{2}, \ldots, s_{m}\right) \in{ }^{k} \mathbf{H}$ to $\left(s_{m}, \ldots, s_{2}, s_{1}\right)$ and thus ${ }^{k} B_{x}$ to ${ }^{k} B_{x^{-1}}$ for all $x \in W$. It is easy to see that $\iota$ descends to a well-known $\mathbb{Z}\left[v, v^{-1}\right]$-linear anti-involution on $\mathcal{H}$ sending $\underline{H}_{x}$ to $\underline{H}_{x^{-1}}$ as well as ${ }^{p} \underline{H}_{x}$ to ${ }^{p} \underline{H}_{x^{-1}}$ for all $x \in W$. By slight abuse of notation we will denote this map also by $\iota$. Thus we have: $\operatorname{ch} \circ \iota=\iota \circ \mathrm{ch}$. Finally expressing ${ }^{p} \underline{H}_{x}$ in the Kazhdan-Lusztig basis and applying $\iota$ proves the claim after comparing coefficients.

(5) The statement for left descent sets follows from Lemma 4.3 below and the fact that the Laurent polynomials ${ }^{p} m_{y, x}$ have non-negative coefficients. Using (4) we can reduce the statement about the right descent sets to the case we have just proven.

(6) This follows immediately from the analogue of Soergel's categorification theorem (part (4) of Theorem 2.4). Indeed, in order to express ${ }^{p} \underline{H}_{x}{ }^{p} \underline{H}_{y}$ in the $p$-canonical basis, we need to decompose ${ }^{k} B_{x}{ }^{k} B_{y}$ into indecomposable objects in ${ }^{k} \mathbf{H}$ and thus the Laurent polynomial ${ }^{p} \mu_{x, y}^{z}$ encodes the graded multiplicity of ${ }^{k} B_{z}$ in this tensor product. Therefore ${ }^{p} \mu_{x, y}^{z}$ has non-negative coefficients. The self-duality of these Laurent polynomials follows from (1).

(7) As explained in Section 3 we need to calculate the graded rank of finitely many local intersection forms in order to calculate ${ }^{p} \underline{H}_{x}$. The rank of each of these intersection forms can only decrease for finitely many primes. (7) now follows.

The multiplication formula from [KL79, (2.3.a) and (2.3.c)]) reads for $x \in W$ and $s \in S$ as follows:

$$
\underline{H}_{s} \underline{H}_{x}= \begin{cases}\left(v+v^{-1}\right) \underline{H}_{x} & \text { if } s x<x, \\ \underline{H}_{s x}+\sum_{\substack{y<x \\ s y<y}} \mu(y, x) \underline{H}_{y} & \text { otherwise. }\end{cases}
$$


where $\mu(y, x)$ is the coefficient of $v$ in the Kazhdan-Lusztig polynomial ${ }^{0} h_{y, x}$. One remnant of this multiplication formula for the $p$-canonical basis is the following result:

Lemma 4.3. For $x \in W$ and $s \in \mathcal{L}(x)$ we have:

$$
{ }^{p} \underline{H}_{s}^{p} \underline{H}_{x}=\left(v+v^{-1}\right)^{p} \underline{H}_{x} .
$$

Proof. Since $s x<x$, we can write using Proposition 4.2 (2) for $\operatorname{ch}\left({ }^{k} B_{s x}\right)$ :

$$
\operatorname{ch}\left({ }^{k} B_{s}{ }^{k} B_{s x}\right)=\underline{H}_{s} \operatorname{ch}\left({ }^{k} B_{s x}\right)=H_{x}+v H_{s x}+\sum_{\substack{y<x \\ y \neq s x}} h_{y} H_{y}=\underline{H}_{x}+\sum_{\substack{y<x \\ y \neq s}} m_{y} \underline{H}_{y} .
$$

As ${ }^{k} B_{x}$ is a summand of ${ }^{k} B_{s}{ }^{k} B_{s x}$ we can apply Proposition 4.2 (3) to deduce

$$
\operatorname{ch}\left({ }^{k} B_{x}\right)=\underline{H}_{x}+\sum_{\substack{y<x \\ y \neq s x}}{ }^{p} m_{y, x} \underline{H}_{y} .
$$

This implies ${ }^{p} m_{s x, x}=0$. Next, we calculate as follows:

$$
\begin{aligned}
{ }^{p} \underline{H}_{s}{ }^{p} \underline{H}_{x} & =\underline{H}_{s}{ }^{p} \underline{H}_{x} \\
& =\underline{H}_{s}\left(\underline{H}_{x}+\sum_{\substack{y<x \\
y \neq s x}}{ }^{p} m_{y, x} \underline{H}_{y}\right) \\
& \in\left(v+v^{-1}\right)^{p} \underline{H}_{x}+\sum_{y<x} \mathbb{Z}_{\geqslant 0}\left[v, v^{-1}\right]^{p} \underline{H}_{y}
\end{aligned}
$$

where in the last equality we used Equation (6) and the observation ${ }^{p} m_{s x, x}=0$ to determine the coefficient in front of ${ }^{p} \underline{H}_{x}$. After evaluating at $v=1$ and acting on the trivial module we see that no other terms besides $\left(v+v^{-1}\right)^{p} \underline{H}_{x}$ can occur on the right hand side.

4.1. The Geometric Satake and the $p$-canonical Basis. In this section, we will explain the consequences of results about parity sheaves on the affine Grassmannian for the $p$-canonical basis.

A very interesting question is under which hypothesis the indecomposable parity sheaves on the affine Grassmannian are perverse. This is equivalent to the $p$-canonical basis being a $\mathbb{Z}$-linear combination of Kazhdan-Lusztig basis elements.

To shorten our notation, write $\mathcal{K}:=\mathbb{C}((t))$ and $\mathcal{O}:=\mathbb{C}[[t]]$. Define the affine Grassmannian $\mathcal{G} r_{a}$ to be the $\mathbb{Z}$-functor given by $R \mapsto$ $L G(R) / L^{+} G(R)$. Its complex points coincide with $G(\mathcal{K}) / G(\mathcal{O})$. For $\lambda \in X^{\vee}$ denote by $t^{\lambda} \in G(\mathcal{K})$ the following composition

$$
\operatorname{Spec}(\mathcal{K}) \longrightarrow \operatorname{Spec}(\mathcal{O})=\mathbb{G}_{m} \stackrel{\lambda}{\longrightarrow} T \hookrightarrow G
$$


where the first morphism comes from the inclusion $\mathcal{O} \hookrightarrow \mathcal{K}$. The Cartan decomposition

$$
\mathcal{G} r_{a}(\mathbb{C})=\bigcup_{\lambda \in X_{+}^{\vee}} \underbrace{G(\mathcal{O}) t^{\lambda} G(\mathcal{O}) / G(\mathcal{O})}_{\mathcal{G} r_{a}^{\lambda}:=}
$$

gives a stratification of $\mathcal{G} r_{a}(\mathbb{C})$ where each stratum $\mathcal{G} r_{a}^{\lambda}$ is a vector bundle over a partial flag variety $G(\mathbb{C}) t^{\lambda} G(\mathcal{O}) / G(\mathcal{O})$. Since the $G(\mathcal{O})$ orbits are all simply-connected, the indecomposable parity sheaves are all parametrized by $\lambda \in X_{+}^{\vee}($ see [JMW14a, Theorem 4.6]):

Theorem 4.4. Assume that the characteristic of $k$ is not a torsion prime ${ }^{4}$ for $G$. For each $\lambda \in X_{+}^{\vee}$ there exists up to isomorphism a unique indecomposable parity complex $\mathcal{E}(\lambda)$ such that $\operatorname{supp}(\mathcal{E}(\lambda))=\overline{\mathcal{G} r_{a}^{\lambda}}$ and $\left.\mathcal{E}(\lambda)\right|_{\mathcal{G} r_{a}^{\lambda}}=\underline{k}_{\mathcal{G} r_{a}^{\lambda}}\left[\operatorname{dim} \mathcal{G} r_{a}^{\lambda}\right]$. Every indecomposable parity complex is isomorphic to $\mathcal{E}(\lambda)$ for some $\lambda \in X_{+}^{\vee}$.

Denote by $P_{G(\mathcal{O})}\left(\mathcal{G} r_{a}(\mathbb{C}), k\right)$ the $G(\mathcal{O})$-equivariant perverse sheaves on $\mathcal{G} r_{a}(\mathbb{C})$ with coefficients in $k$. It comes equipped with a monoidal structure induced by the convolution product *.

Recall that we denote by $\Psi=\left(X, \Phi, X^{\vee}, \Phi^{\vee}\right)$ the root datum of $G$. Define $G^{\vee}$ to be the split connected reductive group scheme over $k$ with root datum (Langlands) dual to that of $G$. To each dominant coweight $\lambda \in X_{+}^{\vee}$ we can associate an induced representation $\nabla(\lambda):=\operatorname{ind}_{B^{\vee}}^{G^{\vee}} k_{\lambda}$, its dual $\Delta(\lambda)$ called the Weyl module and a simple module $L(\lambda)$ sitting in the following sequence $\Delta(\lambda) \rightarrow L(\lambda) \hookrightarrow \nabla(\lambda)$ given by projection to the head and inclusion of the socle. Moreover, the set $\left\{L(\lambda) \mid \lambda \in X_{+}^{\vee}\right\}$ gives a complete set of representatives for the isomorphism classes of simple, rational $G^{\vee}$-modules. The category $\operatorname{Rep}\left(G^{\vee}\right)$ of rational representations of $G^{\vee}$ forms a highest-weight category with Weyl modules as standard objects and induced modules as costandard objects. A rational representation of $G^{\vee}$ is called tilting if it admits two filtrations, one with successive quotients isomorphic to Weyl modules and the other one with successive quotients isomorphic to induced modules. In [Don93, Theorem 1.1] Donkin classifies indecomposable tilting modules for algebraic groups giving in our setting:

Theorem 4.5. For each $\lambda \in X_{+}^{\vee}$ there exists up to isomorphism a unique indecomposable tilting module $T(\lambda)$ of highest weight $\lambda$. Moreover, $\lambda$ occurs with multiplicity one as a weight of $T(\lambda)$. Every indecomposable tilting module is isomorphic to $T(\lambda)$ for some $\lambda \in X_{+}^{\vee}$.

\footnotetext{
${ }^{4}$ See $[J M W 14 a, \S 2.6]$ for the definition of torsion primes. This restriction can be removed by working in the non-equivariant setting.
} 
The Geometric Satake equivalence (see [MV07]) gives a monoidal equivalence

$$
\left(P_{G(\mathcal{O})}\left(\mathcal{G} r_{a}(\mathbb{C}), k\right), *\right) \stackrel{\cong}{\longrightarrow}\left(\operatorname{Rep}\left(G^{\vee}\right), \otimes\right) .
$$

In [JMW14b] Juteau, Mautner and the second author show that if the characteristic $p$ of $k$ is larger than an explicit bound depending on the root system $\Phi$ of $G$, then the indecomposable $k$-parity sheaves on the affine Grassmannian are perverse. More precisely, they show that for $\lambda \in X_{+}^{\vee}$ the indecomposable tilting module $T(\lambda)$ is mapped to $\mathcal{E}(\lambda)$ under the geometric Satake equivalence for $p>b(\Phi)$ (see below).

From now on let us assume that $G$ is adjoint, so $G^{\vee}$ is simply connected. In particular, the cocharacter lattice $X^{\vee}$ coincides with the coweight lattice. Denote by $W_{a}:=W \ltimes \mathbb{Z} \Phi^{\vee}$ the affine Weyl group and by $W_{\text {ext }}:=W \ltimes X^{\vee}$ the extended affine Weyl group. View $W_{a}$ as a Coxeter group $\left(W_{a}, S_{a}\right)$. We can write

$$
W_{\text {ext }}=\Omega \ltimes W_{a}
$$

where $\Omega$ is a finite subgroup of "length zero elements" which acts by automorphisms of the Coxeter system $\left(W_{a}, S_{a}\right)$ (see $[$ Lus83, §2]). The action of $W_{\text {ext }}$ on $X^{\vee}$ gives bijections:

$$
\begin{aligned}
X^{\vee} & \stackrel{\sim}{\longleftrightarrow} W_{\text {ext }} / W, \\
w(0) & \longleftrightarrow \\
\longleftrightarrow & w, \\
X_{+}^{\vee} & \stackrel{\sim}{\longleftrightarrow} W \backslash W_{\text {ext }} / W=\bigcup_{\sigma \in \Omega} \sigma(W) \backslash W_{a} / W .
\end{aligned}
$$

Given $\lambda \in X_{+}^{\vee}$ we denote by $w_{\lambda} \in W_{a}$ the maximal element in the double coset in $\bigcup_{\sigma \in \Omega} \sigma(W) \backslash W_{a} / W$ that corresponds to $\lambda$ under the bijection above. In this section we will work with the Hecke algebra $\mathcal{H}_{a}$ associated to $\left(W_{a}, S_{a}\right)$.

The next results follows because the projection from the affine flag variety to the affine Grassmannian is a stratified fiber bundle with fibers isomorphic to the finite flag variety $\mathcal{F l}$ :

Theorem 4.6. Suppose that all parity sheaves on the affine Grassmannian are perverse. Then ${ }^{p} m_{w_{\lambda}, w_{\nu}} \in \mathbb{Z}$ for all $\lambda, \nu \in X_{+}^{\vee}$.

The explicit bound has later been improved by Mautner and Riche in [MR15]. They show that the parity sheaves on the affine Grassmannian are perverse whenever the characteristic $p$ of $k$ is good for $G$, thus giving the following bounds $p>b(\Phi)$ if the root system $\Phi$ of $G$ is irreducible: 


$$
\begin{array}{c||c|c|c|c}
\text { Type of } \Phi & A_{n} & B_{n}, C_{n}, D_{n} & E_{6}, E_{7}, F_{4}, G_{2} & E_{8} \\
\hline b(\Phi) & 1 & 2 & 3 & 5
\end{array}
$$

The following result gives an interpretation of the multiplicities in the $p$-canonical basis in terms of $\operatorname{Rep}\left(G^{\vee}\right)$ (see $[\mathrm{JMW} 14 \mathrm{~b}$, Corollary 4.1] for the first part):

Lemma 4.7. Suppose that the characteristic $p$ of $k$ satisfies $p>b(\Phi)$. Then we have for $\lambda, \mu \in X_{+}^{\vee}$ :

(1) ${ }^{p} h_{w_{\mu}, w_{\lambda}}(1)=\operatorname{dim} T(\lambda)_{\mu}$,

(2) ${ }^{p} m_{w_{\mu}, w_{\lambda}}=[T(\lambda): \Delta(\mu)]=[T(\lambda): \nabla(\mu)]$ where $[T(\lambda): \Delta(\mu)]$ denotes the multiplicity of $\Delta(\mu)$ in a $\Delta$-flag on $T(\lambda)$.

The first part of the last result shows that ${ }^{p} h_{w_{\mu}, w_{\lambda}}$ gives a $q$-analogue of the weight multiplicity of $\mu$ in $T(\lambda)$. In characteristic 0 (where $T(\lambda)=L(\lambda))$ this result can be found in [Lus83, Theorem 6.1]. In particular, knowledge of the characters of indecomposable tilting modules for $G^{\vee}$ is equivalent to the knowledge of the $p$-canonical basis elements $\left\{{ }^{p} \underline{H}_{w_{\lambda}} \mid \lambda \in X_{+}^{\vee}\right\}$.

\section{Examples}

According to the classification of root systems and connected semisimple algebraic groups, a Dynkin diagram fixes a semi-simple, adjoint algebraic group $G$ together with a maximal torus $T \subseteq G$ such that the root system determined by $(G, T)$ corresponds to the given Dynkin diagram. In this section we will only give the Dynkin diagram and consider the corresponding root datum of this pair $(G, T)$ together with an arbitrary basis labelled by the nodes of the Dynkin diagram as input.

5.1. Type $B_{2}$. We label the simple reflections as follows:

$$
s=t
$$

That means for the pairing between the simple coroots and roots:

$$
\begin{aligned}
& \left\langle\alpha_{t}, \alpha_{s}^{\vee}\right\rangle=-2, \\
& \left\langle\alpha_{s}, \alpha_{t}^{\vee}\right\rangle=-1 .
\end{aligned}
$$

Because the Schubert varieties associated to $e, s, t, s t, t s$ and stst are smooth, we have ${ }^{p} \underline{H}_{x}=\underline{H}_{x}$ for $x \in\{e, s, t, s t, t s, s t s t\}$ and all primes $p$. (This can also be checked directly.) The remaining two elements are sts and tst. The two subsequences of $(s, t, s)$ expressing $s$ and 
corresponding light leaves are:

$$
(U 1, U 0, D 0) \text { of defect } 0 \leadsto L_{1}=
$$

Thus the local intersection form of $(s, t, s)$ at $s$ is given by

$$
I_{s t s, s}=\left(L_{i} \circ \overline{L_{j}}\right)_{i, j \in\{1,2\}}=\left(\begin{array}{cc}
\left\langle\alpha_{t}, \alpha_{s}^{\vee}\right\rangle & \alpha_{t} \\
\alpha_{t} & \alpha_{s} \alpha_{t}
\end{array}\right)
$$

where the top left entry comes from the following calculation:

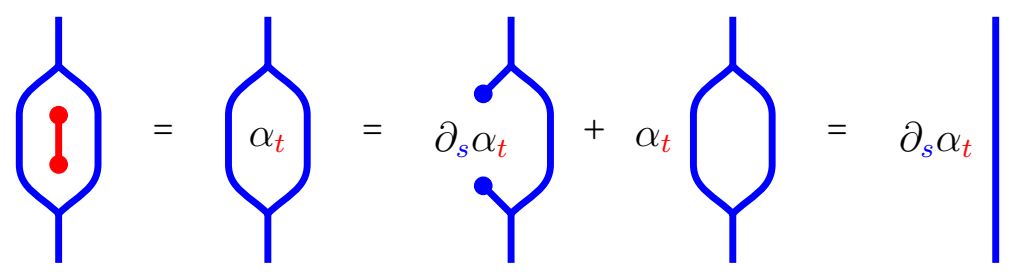

This shows that if $p=2$, then ${ }^{k} B_{s}{ }^{k} B_{t}{ }^{k} B_{s}$ does not decompose as ${ }^{k} B_{s t s} \oplus{ }^{k} B_{s}$, but remains indecomposable. We get

$$
{ }^{p} \underline{H}_{s t s}= \begin{cases}\underline{H}_{s t s}+\underline{H}_{s} & \text { if } p=2, \\ \underline{H}_{s t s} & \text { otherwise. }\end{cases}
$$

Swapping the roles of $s$ and $t$, the same calculation yields

$$
{ }^{p} \underline{H}_{t s t}=\underline{H}_{t s t}
$$

for all primes $p$ as $\left\langle\alpha_{s}, \alpha_{t}^{\vee}\right\rangle=-1$.

Observe that in this case the whole local intersection form of $(s, t, s)$ at $s$ can be calculated using the formula in the nil Hecke ring which we explained in Section 3.1.

5.2. Type $G_{2}$. We label the simple reflections as follows:

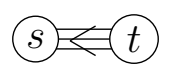

That means for the pairing between the simple coroots and roots:

$$
\begin{aligned}
& \left\langle\alpha_{t}, \alpha_{s}^{\vee}\right\rangle=-3, \\
& \left\langle\alpha_{s}, \alpha_{t}^{\vee}\right\rangle=-1 .
\end{aligned}
$$

For all primes $p>3$ the Kazhdan-Lusztig basis coincides with the $p$-canonical basis. Since the Cartan matrix is symmetric modulo 2, the 2 -canonical basis is stable under swapping $s$ and $t$. Here is a summary 
of the results for $p \in\{2,3\}$ :

$$
\begin{aligned}
{ }^{2} \underline{H}_{s t s t} & =\underline{H}_{s t s t}+\underline{H}_{s t} & { }^{3} \underline{H}_{s t s} & =\underline{H}_{s t s}+\underline{H}_{s} \\
{ }^{2} \underline{H}_{s t s t s} & =\underline{H}_{s t s t s}+\underline{H}_{s} & { }^{3} \underline{H}_{s t s t s} & \underline{H}_{s t s t s}+\underline{H}_{s t s} \\
{ }^{2} \underline{H}_{x} & =\underline{H}_{x} \quad \text { for } & { }^{3} \underline{H}_{x} & =\underline{H}_{x} \text { for } \\
x & \notin\{\text { stst }, \text { tsts, ststs,tstst }\} & x & \notin\{\text { sts, ststs }\}
\end{aligned}
$$

In this example, all the calculations needed to determine ${ }^{p} \underline{H}_{x}$ for $x \notin\{$ ststs, tstst $\}$ can be carried out using the formula in the nil Hecke ring from Section 3.1. For $(s, t, s, t, s)$ (resp. $(t, s, t, s, t))$ there is a subexpression of defect 0 expressing $s$ (resp. $t$ ) that contains a $D 1$ :

$$
(U 1, U 1, U 0, D 1, D 0) \text {. }
$$

We will illustrate how useful the formula in the nil Hecke ring is by calculating the intersection form of $(s, t, s, t)$ at st. For the KazhdanLusztig basis we know:

$$
\underline{H}_{s} \underline{H}_{t} \underline{H}_{s} \underline{H}_{t}=\underline{H}_{s t s t}+2 \underline{H}_{s t} .
$$

There are two subexpressions of $(s, t, s, t)$ of defect 0 expressing st:

$$
\begin{aligned}
& \underline{e}^{1}:=(U 1, U 0, D 0, U 1) \\
& \underline{e}^{2}:=(U 1, U 1, U 0, D 0)
\end{aligned}
$$

We need to calculate the coefficient of $D_{s t}$ in the following elements of the nil Hecke ring:

$$
\begin{array}{ll}
d\left(\underline{e}^{1}, \underline{e}^{1}\right): & D_{s} \alpha_{t} D_{s} D_{t}=\partial_{s}\left(\alpha_{t}\right) D_{s t} \\
d\left(\underline{e}^{1}, \underline{e}^{2}\right): & D_{s} 11 D_{t}=D_{s t} \\
d\left(\underline{e}^{2}, \underline{e}^{2}\right): & D_{s} D_{t} \alpha_{s} D_{t}=\partial_{t}\left(\alpha_{s}\right) D_{s t}
\end{array}
$$

Therefore the local intersection form of stst at st is given by

$$
\left(\begin{array}{cc}
-3 & 1 \\
1 & 1
\end{array}\right)
$$

which implies the result stated above.

5.3. Type $\tilde{A}_{1}$. We label the simple reflections in $S_{a}$ as follows:

$$
\text { (s) } \infty
$$

That means for the pairing between the simple coroots and roots:

$$
\begin{aligned}
& \left\langle\alpha_{t}, \alpha_{s}^{\vee}\right\rangle=-2, \\
& \left\langle\alpha_{s}, \alpha_{t}^{\vee}\right\rangle=-2 .
\end{aligned}
$$


As we discussed in Section 4.1, knowledge of the characters of the tilting modules for $S L_{2}$ in characteristic $p$ gives us part of the $p$-canonical basis for the Weyl group of type $\widetilde{A}_{1}$. Actually in this case two miracles occur:

(1) Donkin's tilting tensor product theorem ([Don93, Proposition 2.1]) together with the knowledge of the characters of the fundamental tilting modules (e.g. see [DH05, Lemma 1.1]) allow us to determine the characters of all indecomposable tilting modules. (This is the only semi-simple group for which all tilting characters are known).

(2) The set $\left\{w_{\lambda} \mid \lambda \in X_{+}^{\vee}\right\}$ together with its image under the automorphism $s \leftrightarrow t$ yields the set $W_{a} \backslash\{$ id $\}$. In particular, knowledge of the tilting characters gives the whole $p$-canonical basis for $S L_{2}$.

To bypass the calculations, the following result (see [EH02, Lemma $6]$ ) is useful because it gives a combinatorial description of the $\Delta$ multiplicities of an indecomposable tilting module.

Lemma 5.1. Let $n \in \mathbb{Z}_{\geqslant 0}$. Write $n$ uniquely as $\sum_{i=0}^{l} n_{i} p^{i}$ with $p-1 \leqslant$ $n_{i} \leqslant 2 p-2$ for $i<l$ and $0 \leqslant n_{l} \leqslant p-1$.

Then $\Delta(m)$ occurs in a $\Delta$-flag of $T(n)$ if and only if $m=\sum_{i=0}^{l} m_{i} p^{i}$ where $m_{j}=n_{j}$ or $m_{j}=2 p-2-n_{j}$ for $j<l$ and $m_{l}=n_{l}$. Moreover, the multiplicity $[T(n): \Delta(m)]$ is at most one.

Observe that in the last lemma for $m=\sum_{i=0}^{l} m_{i} p^{i}$ the coefficients are not required to satisfy $p-1 \leqslant m_{i} \leqslant 2 p-2$ for $i<m$. For $n=\sum_{i=0}^{l} n_{i} p^{i}$ as in the lemma there are precisely $2^{r}$ natural numbers $m$ such that $\Delta(m)$ occurs in a $\Delta$-flag of $T(n)$ where $r=\mid\left\{0 \leqslant i<l \mid n_{i} \neq p-1\right\}$.

To give an example of how this works, consider $n=15$. This is uniquely written as $15=3 \cdot 3^{0}+4 \cdot 3+0 \cdot 3^{2}$ (as described in the last lemma; the last 0 matters!) and thus 4 standard modules occur (with multiplicity one) in a $\Delta$-flag of $T(15)$, namely $\Delta(m)$ for $m$ among the following:

$$
\begin{aligned}
1 & =1 \cdot 3^{0}+0 \cdot 3^{1}+0 \cdot 3^{2}, \\
3 & =3 \cdot 3^{0}+0 \cdot 3^{1}+0 \cdot 3^{2}, \\
13 & =1 \cdot 3^{0}+4 \cdot 3^{1}+0 \cdot 3^{2}, \\
15 & =3 \cdot 3^{0}+4 \cdot 3^{1}+0 \cdot 3^{2} .
\end{aligned}
$$

See Figure 1 at the end of the paper for the multiplicities of $\Delta(m)$ in $T(n)$ for $p=3$ where each black box represents a one. Using Lemma 4.7 
we get for example:

$$
\begin{aligned}
& { }^{3} \underline{H}_{s} \quad=\underline{H}_{s} \\
& { }^{3} \underline{H}_{s t} \quad=\quad \underline{H}_{s t} \\
& { }^{3} \underline{H}_{s t s}=\underline{H}_{s t s} \\
& { }^{3} \underline{H}_{s t s t}=\underline{H}_{s t}+\underline{H}_{s t s t} \\
& { }^{3} \underline{H}_{s t s t s}=\underline{H}_{s}+\underline{H}_{\text {ststs }} \\
& { }^{3} \underline{H}_{\text {ststst }}=\quad \underline{H}_{\text {ststst }} \\
& { }^{3} \underline{H}_{\text {stststs }}=\quad \underline{H}_{\text {ststs }}+\underline{H}_{\text {stststs }} \\
& { }^{3} \underline{H}_{\text {stststst }}=\quad \underline{H}_{\text {stst }}+\underline{H}_{\text {stststst }}
\end{aligned}
$$

Note that even in this relatively simple example one sees a beautiful fractal-like structure emerging!

5.4. Types $B_{3}$ and $C_{3}$. In the Dynkin diagrams of types $B_{3}$ and $C_{3}$ we label the simple reflections as follows:

$$
\begin{aligned}
& B_{3}: 1>2<3 \\
& C_{3}: 1>2
\end{aligned}
$$

The only interesting case is $p=2$. The following table gives an overview over all the Weyl group elements for which the 2-canonical basis differs from the Kazhdan-Lusztig basis. It illustrates the dependence of the 2-canonical basis on the type of the root system. Even 
though the combinatorics in types $B_{3}$ and $C_{3}$ are the same, the corresponding 2-canonical bases are quite different.

\begin{tabular}{|c|c|c|}
\hline & $B_{3}$ & $C_{3}$ \\
\hline${ }^{2} \underline{H}_{212}$ & $\underline{H}_{212}$ & $\underline{H}_{212}+\underline{H}_{2}$ \\
\hline${ }^{2} \underline{H}_{121}$ & $\underline{H}_{121}+\underline{H}_{1}$ & $\underline{H}_{121}$ \\
\hline${ }^{2} \underline{H}_{3212}$ & $\underline{H}_{3212}$ & $\underline{H}_{3212}+\underline{H}_{32}$ \\
\hline${ }^{2} \underline{H}_{2123}$ & $\underline{H}_{2123}$ & $\underline{H}_{2123}+\underline{H}_{23}$ \\
\hline${ }^{2} \underline{H}_{1321}$ & $\underline{H}_{1321}+\underline{H}_{13}$ & $\underline{H}_{1321}$ \\
\hline${ }^{2} \underline{H}_{1213}$ & $\underline{H}_{1213}+\underline{H}_{13}$ & $\underline{H}_{1213}$ \\
\hline${ }^{2} \underline{H}_{32123}$ & $\underline{H}_{32123}$ & $\underline{H}_{32123}+\underline{H}_{232}+\underline{H}_{3}$ \\
\hline${ }^{2} \underline{H}_{21232}$ & $\underline{H}_{21232}$ & $\underline{H}_{21232}+\underline{H}_{232}$ \\
\hline${ }^{2} \underline{H}_{23212}$ & $\underline{H}_{23212}$ & $\underline{H}_{23212}+\underline{H}_{232}$ \\
\hline${ }^{2} \underline{H}_{21321}$ & $\underline{H}_{21321}+\underline{H}_{213}$ & $\underline{H}_{21321}$ \\
\hline${ }^{2} \underline{H}_{12132}$ & $\underline{H}_{12132}+\underline{H}_{132}$ & $\underline{H}_{12132}$ \\
\hline${ }^{2} \underline{H}_{232123}$ & $\underline{H}_{232123}$ & $\underline{H}_{232123}+\left(v+v^{-1}\right) \underline{H}_{232}$ \\
\hline${ }^{2} \underline{H}_{212321}$ & $\underline{H}_{212321}$ & $\underline{H}_{212321}+\underline{H}_{2321}$ \\
\hline${ }^{2} \underline{H}_{121321}$ & $\underline{H}_{121321}+\underline{H}_{1212}+\underline{H}_{1321}+\underline{H}_{1213}+\underline{H}_{13}$ & $\underline{H}_{121321}$ \\
\hline $2 \underline{H}_{123212}$ & $\underline{H}_{123212}$ & $\underline{H}_{123212}+\underline{H}_{1232}$ \\
\hline $2 \underline{H}_{2123212}$ & 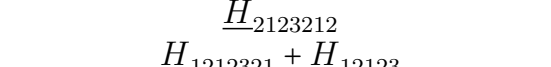 & $\underline{H}_{2123212}+\underline{H}_{21232}+\underline{H}_{23212}+\underline{H}_{232}$ \\
\hline${ }^{2} \underline{I I}_{1} 1212321$ & $\underline{H}_{1212321}+\underline{H}_{12123}$ & $\underline{H}_{H} 1212321$ \\
\hline${ }^{2} \underline{H}_{21232123}$ & $\begin{array}{c}\underline{H}_{1213212}+\underline{H}_{13212} \\
\underline{H}_{21232123}\end{array}$ & $\underline{H}_{21232123} \underline{\underline{I I}}_{1213212} \underline{H}_{232123}$ \\
\hline${ }^{2} \underline{\bar{H}}_{12123212}^{2122120}$ & $\underline{H}_{12123212}+\underline{H}_{1212}$ & $\underline{H}_{12123212}$ \\
\hline${ }^{2} \underline{H}_{12132123}$ & $\underline{H}_{12132123}+\underline{H}_{132123}$ & $\underline{H}_{12132123}$ \\
\hline
\end{tabular}

The most interesting entry in the whole table occurs for type $C_{3}$ and the element $232123 \in W$ where we have

$$
{ }^{2} \underline{H}_{232123}=\underline{H}_{232123}+\left(v+v^{-1}\right) \underline{H}_{232}
$$

This means that, in the decomposition of $B_{232123} \otimes_{\mathbb{O}} \mathbb{K}$ into indecomposable objects in ${ }^{\mathbb{K}} \mathbf{H}$, non-self-dual summands (i.e. with a non-trivial grading shift) occur.

5.5. Type $D_{4}$. We label the simple reflections as follows:

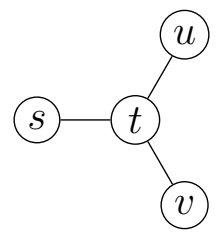

It turns out that the $p$-canonical basis and the Kazhdan-Lusztig basis coincide for all primes except for $p=2$. There are four elements $x \in W$ with ${ }^{2} \underline{H}_{x} \neq \underline{H}_{x}$. If $x=$ suvtsuv then we have

$$
{ }^{2} \underline{H}_{t_{1} x t_{2}}=\underline{H}_{t_{1} x t_{2}}+\underline{H}_{t_{1} \text { suvt }}
$$


for $t_{1}, t_{2} \in\langle t\rangle$. We will give some more details on how to calculate ${ }^{2} \underline{H}_{x}$. We start out by decomposing the corresponding Bott-Samelson object into indecomposable objects in ${ }^{\mathbb{K}} \mathbf{H}$ to get

$$
H_{s} H_{u} H_{v} H_{t} H_{s} H_{u} H_{v}=\underline{H}_{x}+\left(v^{-2}+3+v^{2}\right) \underline{H}_{s u v} .
$$

As subexpressions of $(s, u, v, t, s, u, v)$ expressing $s u v$ we get

$$
\begin{array}{ll}
(U 1, U 1, U 1, U 0, D 0, D 0, D 0) & \text { of defect }-2, \\
(U 1, U 1, U 0, U 0, D 0, D 0, U 1) & \text { of defect } 0, \\
(U 1, U 0, U 1, U 0, D 0, U 1, D 0) & \text { of defect } 0, \\
(U 0, U 1, U 1, U 0, U 1, D 0, D 0) & \text { of defect } 0, \\
(U 1, U 0, U 0, U 0, D 0, U 1, U 1) & \text { of defect 2, } \\
(U 0, U 1, U 0, U 0, U 1, D 0, U 1) & \text { of defect 2, } \\
(U 0, U 0, U 1, U 0, U 1, U 1, D 0) & \text { of defect 2, } \\
(U 0, U 0, U 0, U 0, U 1, U 1, U 1) & \text { of defect } 4 .
\end{array}
$$

The light leaf morphism of degree -2 pairs with the three light leaf morphisms of degree 2 to give the matrix

$$
\left(\begin{array}{l}
-1 \\
-1 \\
-1
\end{array}\right)
$$

The light leaf morphisms corresponding to the subexpressions of defect 0 are the following:

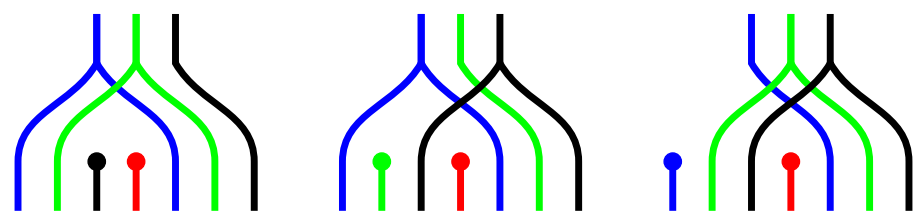

Pairing them gives the following degree 0 piece of the intersection form:

$$
\left(\begin{array}{ccc}
0 & -1 & -1 \\
-1 & 0 & -1 \\
-1 & -1 & 0
\end{array}\right) .
$$

Note that the determinant of this matrix is -2 and its rank in characteristic 2 is 2 . Therefore ${ }^{k} B_{\text {suvtsuv }} \otimes_{\mathbb{O}} \mathbb{K}$ decomposes as

$$
{ }^{\mathbb{K}} B_{\text {suvtsuv }} \oplus^{\mathbb{K}} B_{\text {suv }}
$$


giving the result we stated above. The geometry of this example is discussed in the appendix of [WB12]. Note that all calculations presented in this section can also be carried out using the formula in the nil Hecke ring (see [HW15, §6.2]).

5.6. Type $A_{n}$. According to [WB12], the $p$-canonical basis and the Kazhdan-Lusztig basis coincide for all primes $p$ for $n<7$. Thus, we will describe the case $n=7$ where the situation is quite remarkable.

For all primes $p \neq 2$ the $p$-canonical basis and the Kazhdan-Lusztig basis agree. We have ${ }^{2} \underline{H}_{x} \neq \underline{H}_{x}$ for exactly 38 out of 40320 elements in $S_{8}$ and these examples fall into four classes.

In the following we will denote for a subset $I \subseteq S$ the corresponding parabolic subgroup by $W_{I}=\langle s \in I\rangle \subseteq W$. If $W_{I}$ is finite, its longest element will be denoted by $w_{I}$. A permutation $\phi \in S_{8}$ will be displayed as a string $\phi(1) \phi(2) \ldots \phi(8)$.

The Kashiwara-Saito singularity ([KS97]): This corresponds to the permutation $w=62845173$. We have

$$
{ }^{2} \underline{H}_{w}=\underline{H}_{w}+\underline{H}_{w_{I}}
$$

with $I=\{1,3,4,5,7\}$. There is a cluster of 16 elements around the Kashiwara-Saito singularity described as follows. If we let $J=\{2,6\}$, then we have

$$
{ }^{2} \underline{H}_{u w v}=\underline{H}_{u w v}+\underline{H}_{u w_{I} v} .
$$

for all $u, v \in W_{J}$ unless $u=v=w_{J}$ in which case

$$
{ }^{2} \underline{H}_{w_{J} w w_{J}}=\underline{H}_{w_{J} w w_{J}}+\underline{H}_{w_{J} w_{I} w_{J}}+\underline{H}_{w_{K}}
$$

where $K=\{1,2,3,5,6,7\}$.

The Hexagon singularity (Braden's example in the appendix of [WB12]): Consider the permutation $w=46718235$. We have

$$
{ }^{2} \underline{H}_{w}=\underline{H}_{w}+\underline{H}_{w_{I}}
$$

with $I=\{2,3,5,6\}$. In this case we get a cluster of size 4 . For any $u, v \in\left\langle s_{4}\right\rangle$ we have

$$
{ }^{2} \underline{H}_{u w v}=\underline{H}_{u w v}+\underline{H}_{u w_{I} v}
$$

unless $u=v=s_{4}$ in which case

$$
{ }^{2} \underline{H}_{s_{4} w s_{4}}=\underline{H}_{s_{4} w s_{4}}+\underline{H}_{s_{4} w_{I} s_{4}}+\underline{H}_{w_{K}}
$$

where $K=\{1,3,4,5,7\}$.

For the Kashiwara-Saito singularity and the Hexagon singularity the calculation of the the local intersection form of $\underline{w}$ at $w_{I}$ using the formula in the nil Hecke ring can be found in [HW15, §6.1 and §6.2]. In both cases one can find $\underline{w}$, a reduced expression for $w$, such that this local intersection form is a $1 \times 1$ matrix. 
The waterfall: Consider the permutation $w_{1}=67283415$. Then we have

$$
{ }^{2} \underline{H}_{w_{1} u}=\underline{H}_{w_{1} u}+\underline{H}_{w_{I} u} .
$$

for $I=\{1,2,4,5,6\}$ and $w_{J} \neq u \in W_{J}$ with $J=\{3,7\}$.

Similarly, if we let $w_{2}=57813462$, then we have

$$
{ }^{2} \underline{H}_{v w_{2}}=\underline{H}_{v w_{2}}+\underline{H}_{v w_{I}^{\prime}}
$$

where $I^{\prime}=\{2,3,4,6,7\}$ and $w_{J^{\prime}} \neq v \in W_{J}$ with $J=\{1,5\}$.

The situation becomes more complicated due to the fact that for $u=w_{J}$ and $v=w_{J^{\prime}}$ we have $w_{1} u=v w_{2}=: w$. In this case we get

$$
{ }^{2} \underline{H}_{w}=\underline{H}_{w}+\underline{H}_{w_{I} u}+\underline{H}_{v w_{I}^{\prime}} .
$$

Note that, unlike the Kashiwara-Saito and hexagon permutations discussed above, the clusters containing $w_{1}$ and $w_{2}$ are neither swapped nor fixed by the automorphism $s_{i} \mapsto s_{8-i}$ and thus by applying the graph automorphism one obtains another seven elements for which ${ }^{2} \underline{H}_{x} \neq \underline{H}_{x}$. Hence the two "waterfall" clusters contain 14 elements in total.

The basket: Consider the permutation

$$
w=84627351 .
$$

Then for all $u, v \in\left\langle s_{4}\right\rangle$ one has

$$
{ }^{2} \underline{H}_{u w v}=\underline{H}_{u w v}+\underline{H}_{u w_{I} v}
$$

where $I=\{1,2,3,5,6,7\}$.

\section{References}

[AR13] Pramod N. Achar and Laura Rider, Parity sheaves on the affine Grassmannian and the Mirković-Vilonen conjecture, 2013, arXiv:1305.1684.

[AR14] Pramod N. Achar and Simon Riche, Modular perverse sheaves on flag varieties I: tilting and parity sheaves, 2014, arXiv:1401.7245.

[Ari96] Susumu Ariki, On the decomposition numbers of the Hecke algebra of $G(m, 1, n)$, J. Math. Kyoto Univ. 36 (1996), no. 4, 789-808.

[BBD82] A. A. Beillinson, J. Bernstein, and P. Deligne, Faisceaux pervers, Analysis and topology on singular spaces, I (Luminy, 1981), Astérisque, vol. 100, Soc. Math. France, Paris, 1982, pp. 5-171.

[BL94] Joseph Bernstein and Valery Lunts, Equivariant sheaves and functors, Lecture Notes in Mathematics, vol. 1578, Springer-Verlag, Berlin, 1994.

[dCM09] Mark Andrea A. de Cataldo and Luca Migliorini, The decomposition theorem, perverse sheaves and the topology of algebraic maps, Bull. Amer. Math. Soc. (N.S.) 46 (2009), no. 4, 535-633.

[Del80] Pierre Deligne, La conjecture de Weil. II, Inst. Hautes Études Sci. Publ. Math. (1980), no. 52, 137-252.

[DH05] Stephen Doty and Anne Henke, Decomposition of tensor products of modular irreducibles for $\mathrm{SL}_{2}$, Q. J. Math. 56 (2005), no. 2, 189-207. 
[Don93] Stephen Donkin, On tilting modules for algebraic groups, Math. Z. 212 (1993), no. 1, 39-60.

[EH02] Karin Erdmann and Anne Henke, On Ringel duality for Schur algebras, Math. Proc. Cambridge Philos. Soc. 132 (2002), no. 1, 97-116.

[EK10] Ben Elias and Mikhail Khovanov, Diagrammatics for Soergel categories, Int. J. Math. Math. Sci. (2010), Art. ID 978635, 58.

[Eli13] B. Elias, The two-color Soergel calculus, 2013, arXiv:1308.6611.

[EW12] B. Elias and G. Williamson, Diagrammatics for Coxeter groups and their braid groups, 2012, to appear in Quantum Topology, arXiv:1405.4928.

[EW13] B. Elias and G. Williamson, Soergel calculus, 2013, arXiv:1309.0865.

[EW14] Ben Elias and Geordie Williamson, The Hodge theory of Soergel bimodules, Ann. of Math. (2) 180 (2014), no. 3, 1089-1136.

[FW14] Peter Fiebig and Geordie Williamson, Parity sheaves, moment graphs and the p-smooth locus of Schubert varieties, Ann. Inst. Fourier (Grenoble) 64 (2014), no. 2, 489-536.

[Gör10] Ulrich Görtz, Affine Springer fibers and affine Deligne-Lusztig varieties, Affine flag manifolds and principal bundles, Trends Math., Birkhäuser/Springer Basel AG, Basel, 2010, pp. 1-50.

[Gro99] I. Grojnowski, Affine $\mathfrak{s l}_{p}$ controls the representation theory of the symmetric group and related Hecke algebras, 1999, arXiv:math/9907129v1.

[Hum90] James E. Humphreys, Reflection groups and Coxeter groups, Cambridge Studies in Advanced Mathematics, vol. 29, Cambridge University Press, Cambridge, 1990.

[HW15] Xuhua He and Geordie Williamson, Soergel calculus and Schubert calculus, 2015, arXiv:1502.04914.

[Jam90] Gordon James, The decomposition matrices of $\mathrm{GL}_{n}(q)$ for $n \leq 10$, Proc. London Math. Soc. (3) 60 (1990), no. 2, 225-265.

[JMW14a] Daniel Juteau, Carl Mautner, and Geordie Williamson, Parity sheaves, J. Amer. Math. Soc. 27 (2014), no. 4, 1169-1212.

[JMW14b] Daniel Juteau, Carl Mautner, and Geordie Williamson, Parity sheaves and tilting modules, 2014, to appear in Annales scientifiques de l'École normale supérieure, arXiv:1403.1647.

[KL79] David Kazhdan and George Lusztig, Representations of Coxeter groups and Hecke algebras, Invent. Math. 53 (1979), no. 2, 165-184.

[KL80] David Kazhdan and George Lusztig, Schubert varieties and Poincaré duality, Geometry of the Laplace operator (Proc. Sympos. Pure Math., Univ. Hawaii, Honolulu, Hawaii, 1979), Proc. Sympos. Pure Math., XXXVI, Amer. Math. Soc., Providence, R.I., 1980, pp. 185-203.

[KS97] Masaki Kashiwara and Yoshihisa Saito, Geometric construction of crystal bases, Duke Math. J. 89 (1997), no. 1, 9-36.

[Kum02] Shrawan Kumar, Kac-Moody groups, their flag varieties and representation theory, Progress in Mathematics, vol. 204, Birkhäuser Boston, Inc., Boston, MA, 2002.

[Lam01] T. Y. Lam, A first course in noncommutative rings, second ed., Graduate Texts in Mathematics, vol. 131, Springer-Verlag, New York, 2001.

[Lib08] Nicolas Libedinsky, Sur la catégorie des bimodules de Soergel, J. Algebra 320 (2008), no. 7, 2675-2694. 
[Lib10] Nicolas Libedinsky, Presentation of right-angled Soergel categories by generators and relations, J. Pure Appl. Algebra 214 (2010), no. 12, 2265-2278.

[Lib15] Nicolas Libedinsky, Light leaves and Lusztig's conjecture, Adv. Math. 280 (2015), 772-807.

[LLT96] Alain Lascoux, Bernard Leclerc, and Jean-Yves Thibon, Hecke algebras at roots of unity and crystal bases of quantum affine algebras, Comm. Math. Phys. 181 (1996), no. 1, 205-263.

[Lus80] George Lusztig, Some problems in the representation theory of finite Chevalley groups, The Santa Cruz Conference on Finite Groups (Univ. California, Santa Cruz, Calif., 1979), Proc. Sympos. Pure Math., vol. 37, Amer. Math. Soc., Providence, R.I., 1980, pp. 313-317.

[Lus83] George Lusztig, Singularities, character formulas, and a q-analog of weight multiplicities, Analysis and topology on singular spaces, II, III (Luminy, 1981), Astérisque, vol. 101, Soc. Math. France, Paris, 1983, pp. 208-229.

[Mak15] Ruslan Maksimau, Canonical basis, KLR algebras and parity sheaves, J. Algebra 422 (2015), 563-610.

[MR15] Carl Mautner and Simon Riche, Exotic tilting sheaves, parity sheaves on affine Grassmannians, and the Mirkovic-Vilonen conjecture, 2015, arXiv:1501.07369v2.

[MV07] I. Mirković and K. Vilonen, Geometric Langlands duality and representations of algebraic groups over commutative rings, Ann. of Math. (2) 166 (2007), no. 1, 95-143.

[Rou12] Raphaël Rouquier, Quiver Hecke algebras and 2-Lie algebras, Algebra Colloq. 19 (2012), no. 2, 359-410.

[RW15] Simon Riche and Geordie Williamson, Tilting modules and the $p$ canonical basis, 2015, in preparation.

[Sai90] Morihiko Saito, Decomposition theorem for proper Kähler morphisms, Tohoku Math. J. (2) 42 (1990), no. 2, 127-147.

[SGA70] Schémas en groupes. III: Structure des schémas en groupes réductifs, Séminaire de Géométrie Algébrique du Bois Marie 1962/64 (SGA 3). Dirigé par M. Demazure et A. Grothendieck. Lecture Notes in Mathematics, Vol. 153, Springer-Verlag, Berlin-New York, 1970.

[Soe97] Wolfgang Soergel, Kazhdan-Lusztig-Polynome und eine Kombinatorik für Kipp-Moduln, Represent. Theory 1 (1997), 37-68 (electronic).

[Soe98] Wolfgang Soergel, Combinatorics of Harish-Chandra modules, Representation theories and algebraic geometry (Montreal, PQ, 1997), NATO Adv. Sci. Inst. Ser. C Math. Phys. Sci., vol. 514, Kluwer Acad. Publ., Dordrecht, 1998, pp. 401-412.

[Soe00] Wolfgang Soergel, On the relation between intersection cohomology and representation theory in positive characteristic, J. Pure Appl. Algebra 152 (2000), no. 1-3, 311-335, Commutative algebra, homological algebra and representation theory (Catania/Genoa/Rome, 1998).

[Soe01] Wolfgang Soergel, Langlands' philosophy and Koszul duality, Algebrarepresentation theory (Constanta, 2000), NATO Sci. Ser. II Math. Phys. Chem., vol. 28, Kluwer Acad. Publ., Dordrecht, 2001, pp. 379-414. 
[Soe07] Wolfgang Soergel, Kazhdan-Lusztig-Polynome und unzerlegbare Bimoduln über Polynomringen, J. Inst. Math. Jussieu 6 (2007), no. 3, 501-525.

[Spr82] T. A. Springer, Quelques applications de la cohomologie d'intersection, Bourbaki Seminar, Vol. 1981/1982, Astérisque, vol. 92, Soc. Math. France, Paris, 1982, pp. 249-273.

[Spr89] T. A. Springer, Linear algebraic groups, Algebraic geometry, 4 (Russian), Itogi Nauki i Tekhniki, Akad. Nauk SSSR, Vsesoyuz. Inst. Nauchn. i Tekhn. Inform., Moscow, 1989, Translated from the English, pp. 5-136, 310-314, 315.

[VV11] M. Varagnolo and E. Vasserot, Canonical bases and KLR-algebras, J. Reine Angew. Math. 659 (2011), 67-100.

[VW13] Kari Vilonen and Geordie Williamson, Characteristic cycles and decomposition numbers, Math. Res. Lett. 20 (2013), no. 2, 359-366.

[WB12] Geordie Williamson and Tom Braden, Modular intersection cohomology complexes on flag varieties, Math. Z. 272 (2012), no. 3-4, 697-727.

[Wil12] Geordie Williamson, Some examples of parity sheaves, March 2012, Oberwolfach report for the meeting "Enveloping Algebras and Geometric Representation Theory", available under: http://people.mpimbonn.mpg.de/geordie/GWilliamson-EnvAlg.pdf.

[Wil13] Geordie Williamson, Schubert calculus and torsion explosion, 2013, arXiv:1309.5055.

[Wil14] Geordie Williamson, A reducible characteristic variety in type A, 2014, to appear in the proceedings of Vogan's birthday conference, arXiv:1405.3479.

Max Planck Institute for Mathematics, Vivatsgasse 7, 53111 Bonn E-mail address: ltjensen@mpim-bonn.mpg.de

Max Planck Institute for Mathematics, Vivatsgasse 7, 53111 Bonn E-mail address: geordw@gmail.com

URL: http://people.mpim-bonn.mpg.de/geordie/ 


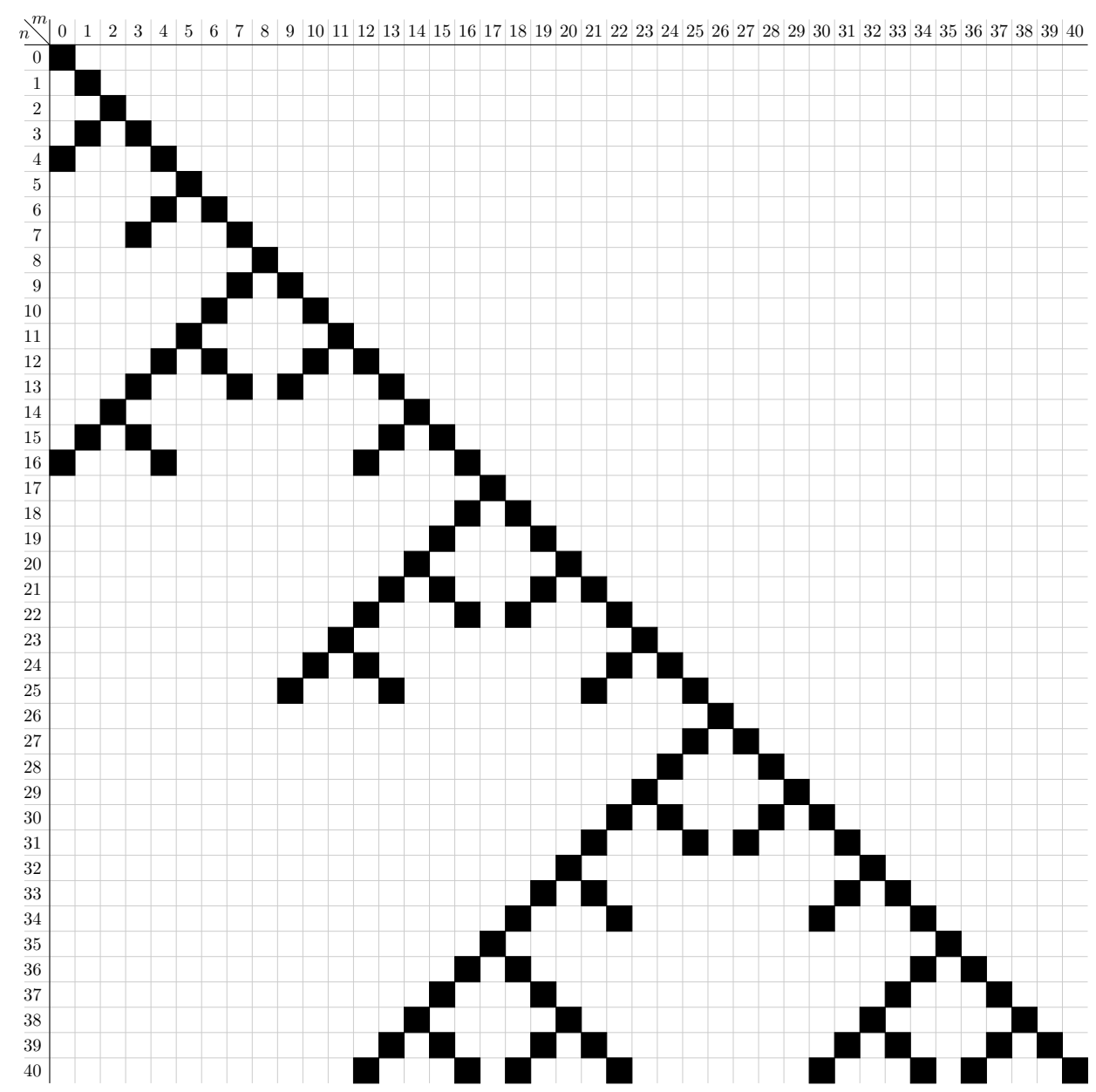

Figure 1. The multiplicities of $\Delta(m)$ in $T(n)$ for $p=3$. 